\title{
Locating the boundary peaks of least-energy solutions to a singularly perturbed Dirichlet problem
}

\author{
TEREsa D’APrile AND JunChEng Wei
}

\begin{abstract}
We consider the problem$$
\varepsilon^{2} \Delta v-v-\gamma_{1} V v+f(v)=0 \quad \Delta V+\gamma_{2}|v|^{2}=0, \quad v=V=0 \text { on } \partial \Omega,
$$

where $\Omega \subset \mathbb{R}^{3}$ is a smooth and bounded domain, $\varepsilon, \gamma_{1}, \gamma_{2}>0, v, V: \Omega \rightarrow \mathbb{R}$, $f: \mathbb{R} \rightarrow \mathbb{R}$. We prove that this system has a least-energy solution $v_{\varepsilon}$ which develops, as $\varepsilon \rightarrow 0^{+}$, a single spike layer located near the boundary, in striking contrast with the result in [37] for the single Schrödinger equation. Moreover the unique peak approaches the most curved part of $\partial \Omega$, i.e., where the boundary mean curvature assumes its maximum. Thus this elliptic system, even though it is a Dirichlet problem, acts more like a Neumann problem for the single-equation case. The technique employed is based on the so-called energy method, which consists in the derivation of an asymptotic expansion for the energy of the solutions in powers of $\varepsilon$ up to sixth order; from the analysis of the main terms of the energy expansion we derive the location of the peak in $\Omega$.
\end{abstract}

Mathematics Subject Classification (2000): 35B40 (primary); 35B45, 35J55, 92C15, 92C40 (secondary).

\section{Introduction}

In this paper we study the following problem:

$$
\begin{cases}\varepsilon^{2} \Delta v-v-\gamma_{1} V v+f(v)=0 & \text { in } \Omega, \\ \Delta V+\gamma_{2} v^{2}=0 & \text { in } \Omega, \\ v, V>0 & \text { in } \Omega, \quad v=V=0 \text { on } \partial \Omega\end{cases}
$$

where $\Omega \subset \mathbb{R}^{3}$ is a smooth and bounded domain, $\varepsilon, \gamma_{1}, \gamma_{2}>0, v, V: \Omega \rightarrow \mathbb{R}$, $f: \mathbb{R} \rightarrow \mathbb{R}$. Solutions of (1.1) correspond to the stationary waves for the following Schrödinger-Poisson system:

$$
i \varepsilon \frac{\partial \psi}{\partial t}=-\varepsilon^{2} \Delta \psi+\psi+\gamma_{1} V \psi-f(\psi), \quad \Delta V+\gamma_{2}|\psi|^{2}=0 .
$$

The research of the second author is supported by an Earmarked Grant from RGC of Hong Kong. Received February 2, 2006; accepted in revised form May 24, 2006. 
This system, first proposed by Benci-Fortunato ([3]) and later studied in [6, 13], can be used as a model in Quantum Mechanics to describe a charged particle interacting with its own electrostatic field. The purpose of this paper is to construct a single spike for the system (1.1) located near the boundary, where by single spike we intend a solution whose shape has the form of a unique peak which becomes highly concentrated when $\varepsilon$ is sufficiently small.

When $\gamma_{2}=0$ we obtain the single Schrödinger equation:

$$
\varepsilon^{2} \Delta v-v+f(v)=0,
$$

for which the existence of single and multiple spike solutions has been extensively studied. Concerning equation (1.2) in a bounded domain with Neumann boundary condition, $\mathrm{Ni}$ and Takagi in $[34,35]$ first proved that for $\varepsilon$ sufficiently small there is a least-energy solution $v_{\varepsilon}$ with the property that $v_{\varepsilon}$ has exactly one maximum point $P_{\varepsilon}$ in $\bar{\Omega}$, and $P_{\varepsilon}$ must be located on $\partial \Omega$ and near the most curved part of the $\partial \Omega$, i.e., $\mathcal{H}\left(P_{\varepsilon}\right) \rightarrow \max _{P \in \partial \Omega} \mathcal{H}(P)$, where $\mathcal{H}(P)$ denotes the mean curvature of the boundary $\partial \Omega$. On the other hand, for equation (1.2) in a bounded domain with Dirichlet boundary conditions, $\mathrm{Ni}$ and Wei in [37] showed that the least-energy solution develops a spike layer at the most centered part of the domain, i.e., $\operatorname{dist}\left(P_{\varepsilon}, \partial \Omega\right) \rightarrow$ $\max _{P \in \Omega} \operatorname{dist}(P, \partial \Omega)$. Since then, there have been many papers looking for higherenergy solutions. More specifically, solutions with multiple boundary peaks as well as multiple interior peaks have been established. It turns out that a general guideline is that while multiple boundary spikes tend to cluster around the critical points of the boundary mean curvature $\mathcal{H}(P)$, the location of the interior spikes is governed by the distance between the peaks as well as from the boundary $\partial \Omega$ (see $[1,2,8,9,10,18,19,25,26,27,28,29,32,33,34,35,36,37,39,40]$ and the references therein). In particular, it was established by Gui and Wei ([28]), that given two arbitrary integers $l_{1}$ and $l_{2}$ there exist solutions for the Neumann problem associated to (1.2) with $l_{1}$ peaks on the boundary and $l_{2}$ peaks in the interior.

Our paper deals with the system (1.1) when $\gamma_{2} \neq 0$ and is in striking contrast with the results for single-equation case (1.2), in particular with the above-quoted paper [37]: indeed we will establish that the least-energy solutions of the Dirichlet problem (1.1) exhibit a concentration behaviour at the boundary. Before going further in the analysis of this phenomenon, let us briefly outline the concentration results already known for the system (1.1). The asymptotic analysis of (1.1) has been started very recently in the papers [14]-[17] and [38]. The radially symmetric case has been investigated in [14, 16] and [38]. In [16] and [38] it is proved that for $1<p<\frac{11}{7}$ there exists a family of positive radial solutions in $\mathbb{R}^{3}$ which concentrates at a sphere. In [14] the concentration on all the boundary $\partial \Omega$ is produced for the problem (1.1) when $\Omega$ is the unit ball of $\mathbb{R}^{3}$. In the other recent papers [15] and [17] multiple interior spikes have been shown to exist for (1.1) in the case of a generic bounded domain $\Omega \subset \mathbb{R}^{3}$ (near the harmonic centers of $\Omega$ ) and for the whole of $\mathbb{R}^{N}$ respectively. However peaked solutions approaching the boundary have not yet been observed for (1.1) neither for $\gamma_{2}=0$ nor in the case $\gamma_{2} \neq 0$. This paper seems to be the first attempt in this line. 
In order to provide the exact formulation of our main result we first enumerate the assumptions on the function $f$ that will be steadily assumed:

(f1) $f \in \mathrm{C}^{2}(\mathbb{R}) ; f(t)=0$ for $t \leq 0$;

(f2) $\frac{f(t)}{t^{3}}$ is nondecresing in $t>0$;

(f3) $f(t)=\mathrm{O}\left(t^{p}\right)$ as $t \rightarrow+\infty$, where $3<p<5$;

(f4) there exists a constants $\theta>4$ such that $0<\theta F(t) \leq f(t) t$ for all $t>0$, where $F(t)=\int_{0}^{t} f(s) \mathrm{d} s$

(f5) the problem in the whole space

$$
\left\{\begin{array}{l}
\Delta w-w+f(w)=0, \quad w>0 \quad \text { in } \mathbb{R}^{3}, \\
w(0)=\max _{x \in \mathbb{R}^{3}} w(x), \quad \lim _{|x| \rightarrow+\infty} w(x)=0,
\end{array}\right.
$$

has a unique solution $w$, which is nondegenerate, i.e., denoting by $L$ the linearized operator

$$
L: H^{2}\left(\mathbb{R}^{3}\right) \rightarrow L^{2}\left(\mathbb{R}^{3}\right), \quad L[u]:=\Delta u-u+f^{\prime}(w) u,
$$

then

$$
\operatorname{Kern}(L)=\operatorname{span}\left\{\frac{\partial w}{\partial x_{1}}, \frac{\partial w}{\partial x_{1}}, \frac{\partial w}{\partial x_{3}}\right\} .
$$

By the well-known result of Gidas, $\mathrm{Ni}$ and Nirenberg ([23]) $w$ is radially symmetric and strictly decreasing in $r=|x|$. Moreover, by classical regularity results, $w \in$ $\mathrm{C}^{2}\left(\mathbb{R}^{3}\right)$ and the following asymptotic behavior holds:

$$
w(r), w^{\prime \prime}(r)=\frac{A}{r} e^{-r}\left(1+\mathrm{O}\left(\frac{1}{r}\right)\right), \quad w^{\prime}(r)=-\frac{A}{r} e^{-r}\left(1+\mathrm{O}\left(\frac{1}{r}\right)\right),
$$

where $A>0$ is a suitable positive constant. Note that assumptions (f1)-(f3) imply

$$
f(t) \leq c_{1}|t|^{3}+c_{2}|t|^{p}, \quad F(t) \leq C_{1}|t|^{4}+C_{2}|t|^{p+1} \quad \forall t \geq 0 .
$$

Typical examples of $f$ satisfying (f1)-(f5) include $f(t)=t_{+}^{p}$ where $3<p<5$. Other nonlinearities can be found in [7]. The uniqueness of $w$ is proved in [31] for the case of power-like $f$; for a general nonlinearity, see [5]. The nondegeneracy condition can be derived from the uniqueness argument (see [34]).

We recall the variational structure of the system (1.1): indeed for every $v \in$ $H_{0}^{1}(\Omega)$ let $(-\Delta)^{-1}\left[v^{2}\right] \in H_{0}^{1}(\Omega)$ be the unique solution of the following problem

$$
\Delta V+v^{2}=0 \text { in } \Omega, \quad V=0 \text { on } \partial \Omega .
$$

Then (1.1) is equivalent to

$$
\begin{cases}\varepsilon^{2} \Delta v-v-\gamma(-\Delta)^{-1}\left[v^{2}\right] v+f(v)=0 & \text { in } \Omega, \\ v>0 & \text { in } \Omega, \quad v=0 \text { on } \partial \Omega,\end{cases}
$$


where $\gamma=\gamma_{1} \gamma_{2}$, and associated to (1.6) is the following energy functional:

$J_{\varepsilon}[v]:=\frac{1}{2} \int_{\Omega}\left(\varepsilon^{2}|\nabla v|^{2}+|v|^{2}\right) \mathrm{d} x+\frac{\gamma}{4} \int_{\Omega}(-\Delta)^{-1}\left[v^{2}\right] v^{2} \mathrm{~d} x-\int_{\Omega} F(v) \mathrm{d} x$.

Our aim is to establish the existence of a least-energy solution $v_{\varepsilon}$ for (1.6) and to show that $v_{\varepsilon}$ exhibits a point-condensation phenomenon as $\varepsilon \rightarrow 0^{+}$. More precisely, when $\varepsilon$ is sufficiently small, $v_{\varepsilon}$ has a single spike centered at a point $P_{\varepsilon}$ located at a distance $(1+\mathrm{o}(1)) \varepsilon \log \frac{1}{\varepsilon}$ from the boundary, while $v_{\varepsilon}$ vanishes everywhere else. Hence the following natural question immediately arises: which part of the boundary are the points $P_{\varepsilon}$ situated near? It is the purpose of this paper to answer this question and to give an accurate description of the profiles of the solutions $v_{\varepsilon}$. Indeed we shall prove that this unique peak must be situated near the most curved part of $\partial \Omega$, i.e. where the boundary mean curvature assumes its maximum; more precisely any limiting point $P_{0}$ of the family $P_{\varepsilon}$ is such that $\mathcal{H}\left(P_{0}\right)$, the mean curvature of $\partial \Omega$ at $P_{0}$, is a maximum value of $\mathcal{H}(P)$ over $\partial \Omega$.

Now we proceed to state our main theorem.

Theorem 1.1. Assume that $\Omega \subset \mathbb{R}^{3}$ is a smooth and bounded domain and that the hypotheses (f1)-(f5) hold. Then for every $\varepsilon>0$ there exists a least-energy solution $v_{\varepsilon} \in H_{0}^{1}(\Omega)$ of (1.6). Furthermore, as $\varepsilon \rightarrow 0^{+}$, $v_{\varepsilon}$ develops a spike near the maxima of the mean curvature; more precisely there exists $P_{\varepsilon} \in \Omega$ such that

(1) $v_{\varepsilon}(x)=w\left(\frac{x-P_{\varepsilon}}{\varepsilon}\right)+o(1)$ uniformly in $\Omega$;

(2) $\operatorname{dist}\left(P_{\varepsilon}, \partial \Omega\right)=(1+\mathrm{o}(1)) \varepsilon \log \frac{1}{\varepsilon}$.

Finally, for every sequence $\varepsilon_{n} \rightarrow 0^{+}$, up to a subsequence,

(3) $P_{\varepsilon_{n}} \rightarrow P_{0} \in \partial \Omega$ where $\mathcal{H}\left(P_{0}\right)=\mathcal{H}_{0}:=\max _{P \in \partial \Omega} \mathcal{H}(P)$.

Remark 1.2. Notice that if, in addition, we assume the existence of a unique global maximum $P_{0}$ of $\mathcal{H}(P)$, Part (3) of Theorem 1.1 holds for all the families $P_{\varepsilon}$, without need to pass to subsequences, and all the waves $v_{\varepsilon}$ concentrate at that point $P_{0}$ as $\varepsilon \rightarrow 0^{+}$.

It is interesting to see how the geometry of the domain determines exactly the location of the spike-layers as well as how this result is in striking contrast with the result in [37] for the single Schrödinger equation (1.2) with Dirichlet boundary condition, in which the least-energy solutions are located at the most centered part of the domain. Furthermore, even among higher-energy solutions, it is also known that there are no positive spike-layers concentrating near the boundary for the Dirichlet problem associated to $(1.2)$ (see $[10,41])$. On the contrary least-energy solutions with a single boundary peak close to the maxima of the mean curvature are known for (1.2) with Neumann conditions. So we are in presence of a Dirichlet problem which acts more like a Neumann problem for the single-equation case. 
To our knowledge, the only other results concerning boundary-concentration occurring for a Dirichlet problem were established for the FitzHugh-Nagumo system in [11] and for changing-sign solutions of an elliptic equation in [12] by Dancer and Yan; however in [11] only the existence of such solutions is proved and the exact boundary limiting points are not determined, while in [12] a special kind of nonlinearity is considered such that the changing-sign solutions are obtained as mountain passes and blow-up at the boundary points which are maxima of the main curvature. Although the result in [12] looks similar to that in our paper, the locations of the peaks for the two problems are different: indeed in [12] the solutions are constructed as approximation of a suitable mountain pass solution for an elliptic problem on a half-space (while our limiting problem (1.3) is in the whole space) and then the distance of the peaks from the boundary is $\mathrm{O}(\varepsilon)$ (and not, as in our case, $\left.\mathrm{O}\left(\varepsilon \log \frac{1}{\varepsilon}\right)\right)$. This paper seems to be the first one that succeeds in locating exactly the boundary spikes for positive solutions of a Dirichlet problem.

The proof of Theorem 1.1 is based on the energy method, i.e., on the derivation of an asymptotic formula for the smallest critical value $J_{\varepsilon}^{*}:=J_{\varepsilon}\left[v_{\varepsilon}\right]$ as $\varepsilon \rightarrow 0^{+}$, in the spirit of $[34,35,37]$. However, here the technique is more complicated since we have to expand the energy up to sixth order. The first object is to apply the Mountain-Pass Lemma to obtain a critical point $v_{\varepsilon}$ of $J_{\varepsilon}$; furthermore we prove that $v_{\varepsilon}$ is actually a least-energy solution of (1.6), by which it is meant that $v_{\varepsilon}$ has the smallest energy $J_{\varepsilon}^{*}$ among all the solutions to (1.6), and $J_{\varepsilon}^{*}$ can be characterized as

$$
J_{\varepsilon}^{*}=\inf _{v \in H_{0}^{1}(\Omega)} \max _{t \geq 0} J_{\varepsilon}[t v] .
$$

Then we show that for $\varepsilon$ sufficiently small $v_{\varepsilon}$ is a single spike solution which is localized in a $\varepsilon$-neighborhood of a maximum point $P_{\varepsilon}$ with $\frac{\operatorname{dist}\left(P_{\varepsilon}, \partial \Omega\right)}{\varepsilon} \rightarrow+\infty$. Next, the critical step is to know the detailed structure of $v_{\varepsilon}$ around $P_{\varepsilon}$. To do this we first use the solution $w$ of the limiting problem (1.4) to construct a family of suitable functions $\tilde{w}_{\varepsilon, P}$ and then prove that the solution $v_{\varepsilon}$ can be obtained as a suitable perturbation of $\tilde{w}_{\varepsilon, P_{\varepsilon}}$. To perform such approximation we make extensive use of the nondegeneracy condition (f5). Once we have obtained the shape of $v_{\varepsilon}$, we have to expand $J_{\varepsilon}\left[v_{\varepsilon}\right]=J_{\varepsilon}^{*}$ up to the order $\mathrm{O}\left(\varepsilon^{6}\right)$. The first term in the expansion formula of $J_{\varepsilon}^{*}$ is given by $I[w] \varepsilon^{3}$, where $I[w]$ is the energy of $w$ :

$I[w]=\frac{1}{2} \int_{\mathbb{R}^{3}}\left(|\nabla w|^{2}+|w|^{2}\right) \mathrm{d} x-\int_{\mathbb{R}^{3}} F(w) \mathrm{d} x=\frac{1}{2} \int_{\mathbb{R}^{3}} f(w) w \mathrm{~d} x-\int_{\mathbb{R}^{3}} F(w) \mathrm{d} x$.

The first correction term in $J_{\varepsilon}^{*}$ contains the distance function $P_{\varepsilon}$ from the boundary. The most delicate part is the computation of the nonlocal term $\int_{\Omega}(-\Delta)^{-1}\left[v_{\varepsilon}^{2}\right] v_{\varepsilon}^{2}$ which is the crucial term for locating the peaks at the boundary: its effects are felt at the order $\varepsilon^{6}$ of the expansion where it interacts with the nonlinear part $\int_{\Omega} F\left(v_{\varepsilon}\right)$ giving rise to a term involving the main curvature. Finally the location of $P_{\varepsilon}$ is determined by using $\tilde{w}_{\varepsilon, P}$ as comparison functions (for suitable $P \in \Omega$ ), i.e., according to the characterization (1.8), we compare $J_{\varepsilon}^{*}$ with $\max _{t \geq 0} J_{\varepsilon}\left[t \tilde{w}_{\varepsilon, P}\right]$; such comparison gives information on the terms in the asymptotic expansion of $J_{\varepsilon}^{*}$, in particular on $\operatorname{dist}\left(P_{\varepsilon}, \partial \Omega\right)$ as well as on which portion of the boundary $P_{\varepsilon}$ approaches to. 
We believe that using the asymptotic expansions derived in this paper it is possible to construct single boundary spikes at nondegenerate critical points of the mean curvature (as in [39]), or at topologically nontrivial critical points of the mean curvature (as in [19]). It may also be possible to show the existence of clustered spikes at a local minimum point of the mean curvature (as in [8] and [29]). Another interesting problem is to study the stability of such solutions. We believe that, as for the single-equation case (see [4]), under some conditions on the exponent $p$ the least-energy solution constructed in this paper should be stable.

The paper is organized as follows. Section 2 is devoted to introduce some notation and preliminaries. In Section 3 we construct the approximated solution $\tilde{w}_{\varepsilon, P}$ and we determine its shape. Section 4 contains the expansion of the functional $J_{\varepsilon}$ on $\tilde{w}_{\varepsilon, P}$ as a function of $\varepsilon$ and $P$. In Section 5 we construct the least-energy solutions $v_{\varepsilon}$ and prove that their shape can be approximated by $\tilde{w}_{\varepsilon}, P_{\varepsilon}$, for suitable $P_{\varepsilon} \in \Omega$, up to a certain order $\varepsilon^{\tau_{\varepsilon}}$; furthermore an upper bound for the critical values $J_{\varepsilon}^{*}$ is derived by using $\tilde{w}_{\varepsilon, P}$ as comparison functions and computing $\max _{t \geq 0} J_{\varepsilon}\left[t \tilde{w}_{\varepsilon, P}\right]$. Finally the proof of Theorem 1.1 is completed in Section 6.

ACKNOWLEDGMENTS. This paper was begun while the first author was visiting The Chinese University of Hong Kong in April 2005. She gratefully acknowledges the hospitality of the Department of Mathematics at CUHK.

\section{Notation}

- Given $A \subset \mathbb{R}^{3}$ an open subset, $L^{p}(A)$ is the usual Lebesgue space endowed with the norm

$$
\|u\|_{L^{p}}^{p}:=\int_{A}|u|^{p} \mathrm{~d} x \text { for } 1 \leq p<+\infty, \quad\|u\|_{\infty}=\sup _{x \in A}|u(x)| .
$$

Furthermore $H_{0}^{1}(A)$ is the usual Sobolev space endowed with the norm

$$
\|u\|_{H^{1}}^{2}=\int_{A}\left(|\nabla u|^{2}+|u|^{2}\right) \mathrm{d} x .
$$

- If $u: \mathbb{R}^{N} \rightarrow \mathbb{R}$ is a radially symmetric function, we will continue to denote by $u$ the real function $r>0 \mapsto u(x)$ with $|x|=r$.

- We will often use the symbol $c$ or $C$ to denote different positive constants independent of $\varepsilon$. The value of $c, C$ is allowed to vary from line to line (and also in the same formula).

- o(1) denotes a vanishing quantity as $\varepsilon \rightarrow 0^{+}$.

- Given $\left\{a_{\varepsilon}\right\}_{\varepsilon>0}$ and $\left\{b_{\varepsilon}\right\}_{\varepsilon>0}$ two family of numbers, we write $a_{\varepsilon}=\mathrm{o}\left(b_{\varepsilon}\right)$ (resp. $\left.a_{\varepsilon}=\mathrm{O}\left(b_{\varepsilon}\right)\right)$ to mean that $\frac{a_{\varepsilon}}{b_{\varepsilon}} \rightarrow 0$ (resp. $\left.\left|a_{\varepsilon}\right| \leq C\left|b_{\varepsilon}\right|\right)$ as $\varepsilon \rightarrow 0^{+}$. 


\section{Preliminaries}

In this section we collect some preliminary results concerning the variational structure of the system (1.6). In particular we recall some well-known facts on the representation formula for the Poisson equation: for a smooth domain there exists a unique Green's function $G(x, z)$ of the Laplace operator with Dirichlet boundary condition (see [30]). Furthermore $G$ is symmetric in $x$ and $z$ and

$$
0<G(x, z)<\frac{1}{4 \pi|x-z|} \quad \forall x, z \in \Omega \times \Omega, x \neq z .
$$

Proposition 2.1. Let $\Omega$ be a smooth and bounded domain of $\mathbb{R}^{3}$. For every $g \in$ $L^{2}(\Omega)$ denote by $(-\Delta)^{-1}[g]$ the unique solution in $H_{0}^{1}(\Omega)$ of

$$
-\Delta \psi=g \text {. }
$$

Then the following representation formula holds:

\section{Furthermore}

$$
(-\Delta)^{-1}[g](x)=\int_{\Omega} G(x, z) g(z) \mathrm{d} z .
$$

a) $\int_{\Omega}(-\Delta)^{-1}[g] h \mathrm{~d} x=\int_{\Omega}(-\Delta)^{-1}[h] g \mathrm{~d} x$ for every $g, h \in L^{2}(\Omega)$;

b) $\left\|(-\Delta)^{-1}[g]\right\|_{\infty} \leq C\|g\|_{L^{2}}$ for every $g \in L^{2}(\Omega)$;

c) $\left\|(-\Delta)^{-1}[g]\right\|_{\infty} \leq \varepsilon^{2}\|g\|_{\infty}+\frac{1}{\varepsilon}\|g\|_{L^{1}}$ for every $g \in L^{\infty}(\Omega)$;

d) the functional $\mathcal{J}: u \in H_{0}^{1}(\Omega) \mapsto \int_{\Omega} u^{2}(-\Delta)^{-1}\left[u^{2}\right] \mathrm{d} x$ is $C^{1}$ and

$$
\left\langle\mathcal{J}^{\prime}[u], v\right\rangle=4 \int_{\Omega} u v(-\Delta)^{-1}\left[u^{2}\right] \mathrm{d} x \quad \forall u, v \in H_{0}^{1}(\Omega) .
$$

Proof. By Lax-Milgram's lemma we get the existence of a unique solution in $H_{0}^{1}(\Omega)$ of (2.2). The representation formula (2.3) holds for $u \in C_{0}^{\infty}(\Omega)$ (see, for example, [22, page 23, Theorem 1]); by density (2.3) can be extended to any $g \in L^{2}(\Omega)$. a) follows immediately from (2.3) and Fubini-Tonelli's theorem. By (2.1) for every $g \in L^{2}(\Omega)$, by using Hölder's inequality, we have

$$
\begin{aligned}
\left|(-\Delta)^{-1}[g](x)\right| & \leq \frac{1}{4 \pi} \int_{\Omega} \frac{|g(z)|}{|z-x|} \mathrm{d} z \\
& \leq \frac{1}{4 \pi}\|g\|_{L^{2}}\left(\int_{|z| \leq 2 \operatorname{diam}(\Omega)} \frac{1}{|z|^{2}} \mathrm{~d} z\right)^{1 / 2} \leq C\|g\|_{L^{2}},
\end{aligned}
$$

while, for $g \in L^{\infty}(\Omega)$,

$$
\begin{aligned}
\left|(-\Delta)^{-1}[g](x)\right| & \leq \frac{1}{4 \pi} \int_{|z-x| \leq \varepsilon} \frac{|g(z)|}{|z-x|} \mathrm{d} z+\frac{1}{4 \pi \varepsilon} \int_{\Omega}|g(z)| \mathrm{d} z \\
& \leq \frac{\|g\|_{\infty}}{4 \pi} \int_{|y| \leq \varepsilon} \frac{1}{|z|} \mathrm{d} z+\frac{1}{4 \pi \varepsilon}\|g\|_{L^{1}}=\frac{\varepsilon^{2}\|g\|_{\infty}}{2}+\frac{1}{4 \pi \varepsilon}\|g\|_{L^{1}}
\end{aligned}
$$

and we obtain b)-c). Part d) is a direct computation. 
In view of d) of Proposition 2.1 the energy functional $J_{\varepsilon}$ defined in (1.7) is of class $C^{1}\left(H_{0}^{1}(\Omega), \mathbb{R}\right)$ and its critical points correspond to the solutions of (1.6). Furthermore $J_{\varepsilon}$ can be rewritten as

$$
J_{\varepsilon}[v]=\frac{1}{2} \int_{\Omega}\left(\varepsilon^{2}|\nabla u|^{2}+u^{2}\right) \mathrm{d} x-\int_{\Omega} F(u) \mathrm{d} x+\frac{\gamma}{4} \int_{\Omega} \int_{\Omega} G(x, z) u^{2}(x) u^{2}(z) \mathrm{d} x \mathrm{~d} z .
$$

\section{Computation of $\tilde{w}_{\mathcal{E}, P}$}

In this section we introduce some suitable approximated solutions and derive some crucial estimates: first set

$$
w_{\varepsilon, P}(x)=w\left(\frac{x-P}{\varepsilon}\right), \quad x, P \in \mathbb{R}^{3} .
$$

Next for every $P \in \mathbb{R}^{3}$ define $\tilde{w}_{\varepsilon, P}$ to be the unique solution of the problem

$$
\varepsilon^{2} \Delta \tilde{w}_{\varepsilon, P}-\tilde{w}_{\varepsilon, P}+f\left(w_{\varepsilon, P}\right)=0 \text { in } \Omega, \quad \tilde{w}_{\varepsilon, P}=0 \text { on } \partial \Omega .
$$

From the comparison principle it is immediate that

$$
0<\tilde{w}_{\varepsilon, P}(x)<w_{\varepsilon, P}(x) \quad \forall x \in \Omega, \forall P \in \mathbb{R}^{3} .
$$

The goal is to obtain an asymptotic expansion of the approximations $\tilde{w}_{\varepsilon, P}$. To this aim some preparations are needed. First define the distance function $d_{P}$ from the boundary $\partial \Omega$ by

$$
d_{P}=\operatorname{dist}(P, \partial \Omega), \quad P \in \mathbb{R}^{3} .
$$

The regularity of $\Omega$ implies that $\partial \Omega$ satisfies the uniform interior and exterior sphere condition; that is, at each point $Q \in \partial \Omega$ there exist two balls $B_{1}, B_{2}$ such that $B_{1} \cap \bar{\Omega}=\{Q\}, B_{2} \cap\left(\mathbb{R}^{3} \backslash \Omega\right)=\{Q\}$, and the radii of the balls $B_{1}$ and $B_{2}$ are bounded from below by a positive constant; taking such constant as $\mu$, we obtain that, set

$$
\Gamma_{\mu}:=\left\{P \in \Omega \mid d_{P} \leq \mu\right\},
$$

for every $P \in \Gamma_{\mu}$ there exists a unique $\Sigma_{P} \in \partial \Omega$ such that $\left|\Sigma_{P}-P\right|=\left|\Sigma_{P}-P^{*}\right|=$ $d_{P}=d_{P^{*}}$, (see, for example, [24], page 355), where $P^{*}=2 \Sigma_{P}-P$ (i.e. $P^{*}$ is the symmetric of $P$ with respect to the tangent plane at $\partial \Omega$ in $\left.\Sigma_{P}\right)$. Notice that by construction, using (1.4),

$$
\begin{gathered}
w_{\varepsilon, P^{*}}(x) \leq C \frac{\varepsilon}{d_{P}} e^{-\frac{\left|x-P^{*}\right|}{\varepsilon}} \leq C \frac{\varepsilon}{d_{P}} e^{-\frac{d_{P}}{\varepsilon}} \quad \forall x \in \Omega, \forall P \in \Gamma_{\mu} . \\
w_{\varepsilon, P}(x) \leq C \frac{\varepsilon}{d_{P}} e^{-\frac{|x-P|}{\varepsilon}} \leq C \frac{\varepsilon}{d_{P}} e^{-\frac{d_{P}}{\varepsilon}} \quad \forall x \in \mathbb{R}^{3} \backslash \Omega, \forall P \in \mathbb{R}^{3} .
\end{gathered}
$$


For every $P \in \Gamma_{\mu}$ let $\mathcal{H}_{1}\left(\Sigma_{P}\right), \mathcal{H}_{2}\left(\Sigma_{P}\right)$ be the principal curvatures of $\partial \Omega$ at $\Sigma_{P}$, so that the mean curvature $\mathcal{H}\left(\Sigma_{P}\right)$ of $\partial \Omega$ at $\Sigma_{P}$ is given by the average:

$$
\mathcal{H}\left(\Sigma_{P}\right)=\frac{\mathcal{H}_{1}\left(\Sigma_{P}\right)+\mathcal{H}_{2}\left(\Sigma_{P}\right)}{2}
$$

We introduce a diffeomorfism which straightens a boundary portion near $\Sigma_{P}$ : consider $T_{P}(x)$ the rotation and translation of coordinates which map $\Sigma_{P}$ in 0 , the inner normal to $\partial \Omega$ at $\Sigma_{P}$ in the positive $\ell_{3}$ coordinate axis and the principal directions corresponding to $\mathcal{H}_{1}\left(\Sigma_{P}\right), \mathcal{H}_{2}\left(\Sigma_{P}\right)$ in the $\ell_{1}, \ell_{2}$ axes. Then $T_{P}(P)=\left(0,0, d_{P}\right)$, $T_{P}\left(P^{*}\right)=\left(0,0,-d_{P}\right)$ and in some neighborhood of 0 the boundary $\partial\left(T_{P} \Omega\right)$ can be represented by

$$
y_{3}=\frac{1}{2} \sum_{i=1,2} \mathcal{H}_{j}\left(\Sigma_{P}\right) y_{i}^{2}+\omega_{P}\left(y^{\prime}\right), \quad \lim _{y^{\prime} \rightarrow 0} \frac{\omega_{P}\left(y^{\prime}\right)}{\left|y^{\prime}\right|^{2}}=0, \text { where } y^{\prime}=\left(y_{1}, y_{2}\right) .
$$

Before providing in Proposition 3.2 the asymptotic expansion of the approximated solutions $\tilde{w}_{\varepsilon, P}$ we state first the following useful result.

Lemma 3.1. Fix $a>0, b \geq 0$. For $P \in \Gamma_{\mu}$ such that $\frac{d_{P}}{\varepsilon}$ is sufficiently large the following holds

$$
\left\|w_{\varepsilon, P^{*}}^{a} \frac{\left|y^{\prime}\right|^{b}}{\varepsilon^{b}}\right\|_{L^{\infty}(\Omega)}+\varepsilon^{-3} \int_{\Omega} w_{\varepsilon, P^{*}}^{a} \frac{\left|y^{\prime}\right|^{b}}{\varepsilon^{b}} \mathrm{~d} x \leq C e^{-\frac{2 a d_{P}}{3 \varepsilon}} \quad\left(y=T_{P}(x)\right) .
$$

Proof. According to (3.3), for $\frac{d_{P}}{\varepsilon}$ sufficiently large we get

$$
\begin{aligned}
& \left\|w_{\varepsilon, P^{*}}^{a}\left|y^{\prime}\right|^{b}\right\|_{L^{\infty}(\Omega)}+\varepsilon^{-3} \int_{\Omega} w_{\varepsilon, P^{*}}^{a}\left|y^{\prime}\right|^{b} \mathrm{~d} x \\
& \leq C e^{-\frac{2 a d_{P}}{3 \varepsilon}}\left(\left\|w_{\varepsilon, P^{*}}^{a / 3}\left|y^{\prime}\right|^{b}\right\|_{L^{\infty}(\Omega)}+\varepsilon^{-3} \int_{\Omega} w_{\varepsilon, P^{*}}^{a / 3}\left|y^{\prime}\right|^{b} \mathrm{~d} x\right) \\
& \leq C \varepsilon^{b} e^{-\frac{2 a d_{P}}{3 \varepsilon}}\left(\left\|w^{a / 3}\left(\frac{y+d_{P} \ell_{3}}{\varepsilon}\right) \frac{\left|y^{\prime}\right|^{b}}{\varepsilon^{b}}\right\|_{L^{\infty}\left(\mathbb{R}^{3}\right)}\right. \\
& \left.\quad+\varepsilon^{-3} \int_{\mathbb{R}^{3}} w^{a / 3}\left(\frac{y+d_{P} \ell_{3}}{\varepsilon}\right) \frac{\left|y^{\prime}\right|^{b}}{\varepsilon^{b}} \mathrm{~d} y\right) \\
& \leq C \varepsilon^{b} e^{-\frac{2 d_{P}}{3 \varepsilon}}\left(\left\|w^{a / 3}(y)\left|y^{\prime}\right|^{b}\right\|_{L^{\infty}\left(\mathbb{R}^{3}\right)}+\int_{\mathbb{R}^{3}} w^{a / 3}(y)\left|y^{\prime}\right|^{b} \mathrm{~d} y\right) \leq C \varepsilon^{b} e^{-\frac{2 a d_{P}}{3 \varepsilon}} .
\end{aligned}
$$


Proposition 3.2. For $P \in \Omega$ such that $\frac{d_{P}}{\varepsilon}$ is sufficiently large the following estimates hold:

i) $\tilde{w}_{\varepsilon, P}(x)=w_{\varepsilon, P}(x)+\mathrm{O}\left(\varepsilon^{4}\right)$ uniformly for $x \in \Omega$ and $P \in \Omega \backslash \Gamma_{\mu}$;

ii) $\tilde{w}_{\varepsilon, P}(x)=w_{\varepsilon, P}(x)-w_{\varepsilon, P *}(x)+\mathrm{O}(\varepsilon) h_{\varepsilon, P}(x)+k_{\varepsilon, P}(x)$ uniformly for $x \in \Omega$ and $P \in \Gamma_{\mu}$ where $h_{\varepsilon, P}$ and $k_{\varepsilon, P}$ solve

$$
\begin{gathered}
\varepsilon^{2} \Delta h_{\varepsilon, P}-h_{\varepsilon, P}=0 \text { in } \Omega, \quad h_{\varepsilon, P}=w_{\varepsilon, P^{*}} \frac{\left|y^{\prime}\right|^{2}}{\varepsilon^{2}}+\varepsilon^{4} \text { on } \partial \Omega, \quad y=T_{P}(x), \\
\varepsilon^{2} \Delta k_{\varepsilon, P}-k_{\varepsilon, P}=-f\left(w_{\varepsilon, P^{*}}\right), \quad k_{\varepsilon, P}=0 \text { on } \partial \Omega
\end{gathered}
$$

\section{Furthermore}

$$
\begin{aligned}
& \left\|h_{\varepsilon, P}\right\|_{\infty}^{2}+\varepsilon^{-3} \int_{\Omega} h_{\varepsilon, P}^{2} \mathrm{~d} x=\mathrm{O}\left(\varepsilon^{8}+e^{-\frac{4 d_{P}}{3 \varepsilon}}\right) \\
& \left\|k_{\varepsilon, P}\right\|_{\infty}^{2}+\varepsilon^{-3} \int_{\Omega} k_{\varepsilon, P}^{2} \mathrm{~d} x=\mathrm{O}\left(e^{-\frac{4 d_{P}}{\varepsilon}}\right)
\end{aligned}
$$

uniformly for $P \in \Gamma_{\mu}$.

iii) $\varepsilon \nabla \tilde{w}_{\varepsilon, P}(P)=\mathrm{O}\left(\varepsilon^{4}+\varepsilon e^{-\frac{2 d P}{3 \varepsilon}}+e^{-\frac{2 d_{P}}{\varepsilon}}\right)$.

Proof. The proof of Part i) is immediate: indeed $w_{\varepsilon, P}-\tilde{w}_{\varepsilon, P}$ satisfies

$$
\varepsilon^{2} \Delta\left(w_{\varepsilon, P}-\tilde{w}_{\varepsilon, P}\right)-\left(w_{\varepsilon, P}-\tilde{w}_{\varepsilon, P}\right)=0 \text { in } \Omega, \quad w_{\varepsilon, P}-\tilde{w}_{\varepsilon, P}=w_{\varepsilon, P} \text { on } \partial \Omega .
$$

On the other hand by the definition of $\Gamma_{\mu} w_{\varepsilon, P} \leq C e^{-\frac{\mu}{\varepsilon}}=\mathrm{O}\left(\varepsilon^{4}\right)$ uniformly for $x \in \partial \Omega$ and $P \in \Omega \backslash \Gamma_{\mu}$. The maximum principle implies $w_{\varepsilon, P}-\tilde{w}_{\varepsilon, P}=\mathrm{O}\left(\varepsilon^{4}\right)$ uniformly for $x \in \Omega$ and $P \in \Omega \backslash \Gamma_{\mu}$.

We go on with the proof of Part ii), which is more technical. During its proof it is understood, even though not stated plainly, that all the estimates hold uniformly for $P \in \Gamma_{\mu}$. First decompose

$$
\tilde{w}_{\varepsilon, P}=w_{\varepsilon, P}-w_{\varepsilon, P^{*}}-\varepsilon \hat{h}_{\varepsilon}+k_{\varepsilon}
$$

where $\hat{h}_{\varepsilon}$ solves the following problem

$$
\varepsilon^{2} \Delta \hat{h}_{\varepsilon}-\hat{h}_{\varepsilon}=0 \text { in } \Omega, \quad \hat{h}_{\varepsilon}=\frac{w_{\varepsilon, P}-w_{\varepsilon, P^{*}}}{\varepsilon} \text { on } \partial \Omega,
$$

and $k_{\varepsilon}=k_{\varepsilon, P}$ solves (3.7).

The first object is to prove the following estimate for the boundary points:

$$
\frac{\left|w_{\varepsilon, P}(x)-w_{\varepsilon, P^{*}}(x)\right|}{\varepsilon} \leq C w_{\varepsilon, P^{*}}(x) \frac{\left|y^{\prime}\right|^{2}}{\varepsilon^{2}}+\varepsilon^{4}
$$


uniformly for $x \in \partial \Omega$. Indeed

$$
\begin{aligned}
& d_{P} \leq|x-P|=\left|y-d_{P} \ell_{3}\right|=\sqrt{d_{P}^{2}+|y|^{2}-2 d_{P} y_{3}} \geq\left|y^{\prime}\right|, \\
& d_{P} \leq\left|x-P^{*}\right|=\left|y+d_{P} \ell_{3}\right|=\sqrt{d_{P}^{2}+|y|^{2}+2 d_{P} y_{3}} \geq\left|y^{\prime}\right|
\end{aligned}
$$

uniformly for $x \in \partial \Omega$. Using (3.5) we have $y_{3}=\mathrm{O}\left(\left|y^{\prime}\right|^{2}\right)$ on $\partial \Omega$; consequently

$$
\begin{aligned}
|| x-P|-| x-P^{*}|| & =\frac{|| x-\left.P\right|^{2}-\left|x-P^{*}\right|^{2} \mid}{|x-P|+\left|x-P^{*}\right|} \\
& =\frac{4 d_{P}\left|y_{3}\right|}{|x-P|+\left|x-P^{*}\right|} \leq 2\left|y_{3}\right| \leq C\left|y^{\prime}\right|^{2}
\end{aligned}
$$

uniformly for $x \in \partial \Omega$. Take $x \in \partial \Omega$ and distinguish two cases: first assume $\left|y^{\prime}\right| \geq \sqrt{\varepsilon}$; then, by (3.10), $|x-P|,\left|x-P^{*}\right| \geq \sqrt{\varepsilon}$, and, by (1.4),

$$
\left|w_{\varepsilon, P}(x)\right|,\left|w_{\varepsilon, P^{*}}(x)\right| \leq C \sqrt{\varepsilon} e^{-\frac{1}{\sqrt{\varepsilon}}} \leq \varepsilon^{5} .
$$

Next assume $\left|y^{\prime}\right| \leq \sqrt{\varepsilon}$; then for every $r \in\left[\min \left\{|x-P|,\left|x-P^{*}\right|\right\}, \max \{\mid x-\right.$ $\left.\left.P|| x-,P^{*} \mid\right\}\right]$ by (3.11) we have $\frac{|r-| x-P^{*}||}{\varepsilon} \leq C$, by which, using again (1.4),

$$
\left|w^{\prime}\left(\begin{array}{l}
r \\
\varepsilon
\end{array}\right)\right| \leq C \frac{\varepsilon}{r} e^{-\frac{|r|}{\varepsilon}} \leq C \frac{\varepsilon}{\left|x-P^{*}\right|} e^{-\frac{\left|x-P^{*}\right|}{\varepsilon}} \leq C w_{\varepsilon, P^{*}}(x) ;
$$

hence, by applying the mean value theorem, we get

$$
\left|w_{\varepsilon, P}(x)-w_{\varepsilon, P^{*}}(x)\right| \leq C w_{\varepsilon, P^{*}}(x) \frac{\left\|x-P|-| x-P^{*}\right\|}{\varepsilon} \leq C w_{\varepsilon, P^{*}}(x) \frac{\left|y^{\prime}\right|^{2}}{\varepsilon}
$$

uniformly for $x \in \partial \Omega$ with $\left|y^{\prime}\right| \leq \sqrt{\varepsilon}$. Hence (3.9) holds. The maximum principle applies and gives $\left|\hat{h}_{\varepsilon}\right| \leq C h_{\varepsilon}$ where $h_{\varepsilon}:=h_{\varepsilon, P}$.

By multiplying both members of (3.6) by $h_{\varepsilon}(x)-w_{\varepsilon, P^{*}}(x) \frac{\left|y^{\prime}\right|^{2}}{\varepsilon^{2}}-\varepsilon^{4}$ and since, using (1.4),

$$
\left|\nabla\left(w_{\varepsilon, P^{*}}(x) \frac{\left|y^{\prime}\right|^{2}}{\varepsilon^{2}}\right)\right| \leq \frac{C}{\varepsilon} w_{\varepsilon, P^{*}}(x)\left(1+\frac{\left|y^{\prime}\right|^{2}}{\varepsilon^{2}}\right)
$$


integrating by parts we get

$$
\begin{aligned}
\int_{\Omega}\left(\varepsilon^{2}\left|\nabla h_{\varepsilon}\right|^{2}+h_{\varepsilon}^{2}\right) \mathrm{d} x \leq & C \int_{\Omega} w_{\varepsilon, P^{*}}\left(1+\frac{\left|y^{\prime}\right|^{2}}{\varepsilon^{2}}\right)\left(\varepsilon\left|\nabla h_{\varepsilon}\right|+h_{\varepsilon}\right) \mathrm{d} x+\varepsilon^{4} \int_{\Omega} h_{\varepsilon} \mathrm{d} x \\
\leq & C\left(\int_{\Omega} w_{\varepsilon, P^{*}}^{2}\left(1+\frac{\left|y^{\prime}\right|^{2}}{\varepsilon^{2}}\right)^{2} \mathrm{~d} x\right)^{1 / 2}\left(\int_{\Omega}\left(\varepsilon^{2}\left|\nabla h_{\varepsilon}\right|^{2}+h_{\varepsilon}^{2}\right)\right)^{1 / 2} \\
& +C \varepsilon^{4}\left(\int_{\Omega} h_{\varepsilon}^{2}\right)^{1 / 2} \\
\leq & C \varepsilon^{3 / 2} e^{-\frac{2 d_{P}}{3 \varepsilon}}\left(\int_{\Omega}\left(\varepsilon^{2}\left|\nabla h_{\varepsilon}\right|^{2}+h_{\varepsilon}^{2}\right) \mathrm{d} x\right)^{1 / 2} \\
& +C \varepsilon^{4}\left(\int_{\Omega} h_{\varepsilon}^{2}\right)^{1 / 2}
\end{aligned}
$$

where, in the last inequality, we have used Lemma 3.1. In the same way, by multiplying both members of (3.7) by $k_{\varepsilon}$, since by (1.5) $f(w) \leq C w^{3}$, we have

$$
\int_{\Omega}\left(\varepsilon^{2}\left|\nabla k_{\varepsilon}\right|^{2}+k_{\varepsilon}^{2}\right) \mathrm{d} x \leq C \int_{\Omega} w_{\varepsilon, P^{*}}^{3} k_{\varepsilon} \mathrm{d} x \leq C \varepsilon^{3 / 2} e^{-\frac{2 d_{P}}{\varepsilon}}\left(\int_{\Omega} k_{\varepsilon}^{2} \mathrm{~d} x\right)^{1 / 2}
$$

and a first part of (3.8) follows. In order to complete the proof, first notice that by the maximum principle we derive $h_{\varepsilon}, k_{\varepsilon} \geq 0$ in $\Omega$. Furthermore according to Lemma $3.1 w_{\varepsilon, P} * \frac{\left|y^{\prime}\right|^{2}}{\varepsilon^{2}} \leq C e^{-\frac{2 d P}{3 \varepsilon}}$ on $\partial \Omega$, hence from the maximum principle, $\left\|h_{\varepsilon, P}\right\|_{\infty}=\mathrm{O}\left(e^{-\frac{2 d_{P}}{3 \varepsilon}}+\varepsilon^{4}\right)$. In the same way $f\left(w_{\varepsilon, P^{*}}\right) \leq C w_{\varepsilon, P^{*}}^{3} \leq C e^{-\frac{2 d_{P}}{\varepsilon}}$ on $\Omega$, then the comparison principle gives $\left\|k_{\varepsilon, P}\right\|_{\infty}=\mathrm{O}\left(e^{-\frac{2 d_{P}}{\varepsilon}}\right)$.

Finally to prove Part iii), observe that $z_{\varepsilon, P}=\left(\varepsilon^{4}+\varepsilon e^{-\frac{2 d_{P}}{3 \varepsilon}}+e^{-\frac{2 d_{P}}{\varepsilon}}\right)^{-1}(w-$ $\left.\tilde{w}_{\varepsilon, P}(\varepsilon x+P)\right)$ solves

$$
\Delta z_{\varepsilon, P}=z_{\varepsilon, P} \text { in } B(0,1)
$$

(note that for $\frac{d_{P}}{\varepsilon}$ is sufficiently large we have $B(0,1) \subset \frac{\Omega-P}{\varepsilon}$ ). Furthermore by Parts i)-ii) it follows that $z_{\varepsilon, P}$ is uniformly bounded on $B(0,1)$ (note that $w_{\varepsilon, P^{*}}(\varepsilon x+$ $P) \leq C e^{-\frac{\left|P-P^{*}\right|}{\varepsilon}} \leq C e^{-2 \frac{d P}{\varepsilon}}$ on $\left.B(0,1)\right)$. Then from the well-known Schauder interior estimate $z_{\varepsilon, P}$ and its first and second derivatives are uniformly bounded on the compact sets of $B(0,1)$. Then iii) follows.

An easy consequence of Proposition 3.2 is the following corollary.

Corollary 3.3. Setting $\tilde{w}_{\varepsilon, P}=0$ for $x \notin \Omega$, we have $\tilde{w}_{\varepsilon, P}(\varepsilon x+P) \rightarrow w$ in $H^{1}\left(\mathbb{R}^{3}\right)$ and $L^{\infty}\left(\mathbb{R}^{3}\right)$ as $\frac{d_{P}}{\varepsilon} \rightarrow+\infty$ uniformly for $P \in \Omega$. 
Proof. First observe that $\left\|w_{\varepsilon, P}(\varepsilon x)\right\|_{H^{1}\left(\mathbb{R}^{3} \backslash \frac{\Omega}{\varepsilon}\right)},\left\|w_{\varepsilon, P}(\varepsilon x)\right\|_{L^{\infty}\left(\mathbb{R}^{3} \backslash \frac{\Omega}{\varepsilon}\right)} \rightarrow 0^{+}$as $\frac{d_{P}}{\varepsilon} \rightarrow+\infty$ uniformly for $P \in \Omega$. On the other hand for $P \in \Gamma_{\mu}$, since $\frac{d_{P^{*}}}{\varepsilon}=\frac{d_{P}}{\varepsilon}$,

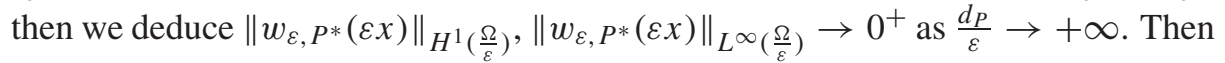
by i) and ii) of Proposition 3.2 this implies that $\tilde{w}_{\varepsilon, P}(\varepsilon x)-w_{\varepsilon, P}(\varepsilon x) \rightarrow 0$ in $L^{2}\left(\mathbb{R}^{3}\right)$ and $L^{\infty}\left(\mathbb{R}^{3}\right)$ as $\frac{d P}{\varepsilon} \rightarrow+\infty$ uniformly for $P \in \Omega$. By multiplying equation (3.1) by $\tilde{w}_{\varepsilon, P}$ and integrating by parts we get

$$
\left\|\tilde{w}_{\varepsilon, P}(\varepsilon x)\right\|_{H^{1}\left(\mathbb{R}^{3}\right)}^{2}=\int_{\mathbb{R}^{3}} f(w) \tilde{w}_{\varepsilon, P}(\varepsilon x+P) \mathrm{d} x \rightarrow \int_{\mathbb{R}^{3}} f(w) w \mathrm{~d} x=\|w\|_{H^{1}\left(\mathbb{R}^{3}\right)}^{2},
$$

by which $\tilde{w}_{\varepsilon, P}(\varepsilon x+P) \rightarrow w$ in $H^{1}\left(\mathbb{R}^{3}\right)$ as $\frac{d_{P}}{\varepsilon} \rightarrow+\infty$ uniformly for $P \in \Omega$.

Our next lemma provides an estimate of the error up to $\tilde{w}_{\varepsilon, P}$ satisfies the system (1.6). To this aim set

$$
\mathcal{S}_{\varepsilon}[v]=\varepsilon^{2} \Delta v-v-\gamma(-\Delta)^{-1}\left[v^{2}\right] v+f(v), \quad v \in H^{2}(\Omega) .
$$

Lemma 3.4. For $P \in \Omega$ such that $\frac{d_{P}}{\varepsilon}$ is sufficiently large the following holds:

$$
\left|\mathcal{S}_{\varepsilon}\left[\tilde{w}_{\varepsilon, P}\right]\right| \leq C\left(e^{-\frac{7 d P}{4 \varepsilon}}+\varepsilon e^{-\frac{2 d P}{3 \varepsilon}}+\varepsilon^{2}\right) w_{\varepsilon, P}^{1 / 4}
$$

Proof. According to c) of Proposition 2.1 and (3.2)

$$
\left|(-\Delta)^{-1}\left[\tilde{w}_{\varepsilon, P}^{2}\right] \tilde{w}_{\varepsilon, P}\right| \leq \varepsilon^{2}\left(\left\|w_{\varepsilon, P}^{2}\right\|_{L^{\infty}(\Omega)}+\varepsilon^{-3}\left\|w_{\varepsilon, P}^{2}\right\|_{L^{1}(\Omega)}\right) w_{\varepsilon, P} \leq C \varepsilon^{2} w_{\varepsilon, P} .
$$

We just need to estimate the local term: by (3.1) and assumption (f1) we deduce

$$
\left|\varepsilon^{2} \Delta \tilde{w}_{\varepsilon, P}-\tilde{w}_{\varepsilon, P}+f\left(\tilde{w}_{\varepsilon, P}\right)\right|=\left|f\left(\tilde{w}_{\varepsilon, P}\right)-f\left(w_{\varepsilon, P}\right)\right| \leq C w_{\varepsilon, P}\left(w_{\varepsilon, P}-\tilde{w}_{\varepsilon, P}\right) .
$$

If $P \in \Omega \backslash \Gamma_{\mu}$ the thesis follows from Part i) of Proposition 3.2. Now assume $P \in \Gamma_{\mu}$; By using Part ii) of Proposition 3.2 we get

$$
\begin{aligned}
\left|\varepsilon^{2} \Delta \tilde{w}_{\varepsilon, P}-\tilde{w}_{\varepsilon, P}+f\left(\tilde{w}_{\varepsilon, P}\right)\right| & \leq C w_{\varepsilon, P}\left(w_{\varepsilon, P^{*}}+\varepsilon h_{\varepsilon, P}+k_{\varepsilon, P}\right) \\
& \leq C w_{\varepsilon, P}\left(w_{\varepsilon, P^{*}}+\varepsilon e^{-\frac{2 d_{P}}{3 \varepsilon}}+\varepsilon^{4}+e^{-\frac{2 d_{P}}{\varepsilon}}\right) .
\end{aligned}
$$

In order to conclude by (1.4) and (3.3) we compute

$$
\begin{aligned}
w_{\varepsilon, P} w_{\varepsilon, P^{*}} & \leq C w_{\varepsilon, P}^{1 / 4} e^{-3 \frac{|x-P|}{4 \varepsilon}-3 \frac{\left|x-P^{*}\right|}{4 \varepsilon}} e^{-\frac{d_{P}}{4 \varepsilon}} \\
& \leq C w_{\varepsilon, P}^{1 / 4} e^{-3 \frac{\left|P-P^{*}\right|}{4 \varepsilon}} e^{-\frac{d_{P}}{4 \varepsilon}}=C w_{\varepsilon, P}^{1 / 4} e^{-\frac{7 d_{P}}{4 \varepsilon}}
\end{aligned}
$$

uniformly for $x \in \Omega$ and $P \in \Gamma_{\mu}$. 


\section{Expansion of $J_{\varepsilon}\left[\tilde{w}_{\varepsilon, p}\right]$}

This section is devoted to compute the energy of the approximated solutions $\tilde{w}_{\varepsilon, P}$. Since the computations are quite long and technical, for the sake of simplicity we provide the expansion for the internal energy and the nonlocal term $\int_{\Omega}(-\Delta)^{-1}\left[\tilde{w}_{\varepsilon, P}^{2}\right] \tilde{w}_{\varepsilon, P}^{2}$ separately in the next Propositions 4.3 and 4.4 respectively. First we need the auxiliary results provided by the following two lemmas.

Lemma 4.1. For $P \in \Gamma_{\mu}$ such that $\frac{d_{P}}{\varepsilon}$ is sufficiently large the following holds

$$
\int_{\Omega} w_{\varepsilon, P}^{a} w_{\varepsilon, P^{*}}^{b} \frac{\left|y^{\prime}\right|^{c}}{\varepsilon^{c}} \mathrm{~d} x \begin{cases}\leq C \varepsilon^{3}\left(\frac{d_{P}}{\varepsilon}\right)^{\frac{c-a}{2}-b+1} e^{-2 b \frac{d_{P}}{\varepsilon}} & \text { if } a>b>0, c \geq 0, \\ \leq C \varepsilon^{3}\left(\frac{d_{P}}{\varepsilon}\right)^{\frac{c-3 a+3}{2}} e^{-2 a \frac{d_{P}}{\varepsilon}} & \text { if } a=b>0, c \geq 0, \\ \leq C \varepsilon^{3}\left(\frac{d_{P}}{\varepsilon}\right)^{\frac{c-a+3}{2}-b} e^{-(a+b) \frac{d_{P}}{\varepsilon}} & \text { if } 0<a<b, c \geq 0,\end{cases}
$$

where $y=T_{P}(x)$.

Proof. By using (1.4) and (3.3) we get

$$
\begin{aligned}
\int_{\Omega} w_{\varepsilon, P}^{a} w_{\varepsilon, P}^{b} \frac{\left|y^{\prime}\right|^{c}}{\varepsilon^{c}} \mathrm{~d} x & \leq C \frac{\varepsilon^{b}}{d_{P}^{b}} \int_{\mathbb{R}^{3}} w^{a}\left(\frac{y-d_{P} \ell_{3}}{\varepsilon}\right) e^{-b \frac{\left|y+d_{P} \ell_{3}\right|}{\varepsilon}} \frac{\left|y^{\prime}\right|^{c}}{\varepsilon^{c}} \mathrm{~d} y \\
& \leq C \frac{\varepsilon^{3+b}}{d_{P}^{b}} \int_{\mathbb{R}^{3}} w^{a}(y) e^{-b\left|y+\frac{2 d_{P} \ell_{3}}{\varepsilon}\right|}\left|y^{\prime}\right|^{c} \mathrm{~d} y \\
& \leq C \frac{\varepsilon^{3+b}}{d_{P}^{b}} \int_{\mathbb{R}^{3}} e^{-a|y|} e^{-b\left|y+2 \frac{d_{P}}{\varepsilon} \ell_{3}\right|}\left|y^{\prime}\right|^{(c-a)_{+}} \mathrm{d} y
\end{aligned}
$$

where $(c-a)_{+}=\max \{0, c-a\}$. Now observe that

$\left|y+2 \frac{d_{P}}{\varepsilon} \ell_{3}\right|=\sqrt{|y|^{2}+4 \frac{d_{P}^{2}}{\varepsilon^{2}}+4 y_{3} \frac{d_{P}}{\varepsilon}} \geq \frac{2 d_{P}}{\varepsilon}+\frac{|y|^{2}}{4 d_{P}} \varepsilon+y_{3} \geq \frac{2 d_{P}}{\varepsilon}+\frac{|y|^{2}}{4 d_{P}} \varepsilon-\left|y_{3}\right|$,

by which

$\int_{\mathbb{R}^{3}} e^{-a|y|} e^{-b\left|y+2 \frac{d_{P}}{\varepsilon} \ell_{3}\right|}\left|y^{\prime}\right|^{(c-a)+} \mathrm{d} y \leq e^{-2 b \frac{d_{P}}{\varepsilon}} \int_{\mathbb{R}^{3}} e^{-a|y|+b\left|y_{3}\right|} e^{-b \frac{|y|^{2}}{4 d_{P}} \varepsilon}\left|y^{\prime}\right|^{(c-a)+} \mathrm{d} y$.

Now we distinguish the three cases: first assume $a>b$; then

$$
\begin{gathered}
\int_{\mathbb{R}^{3}} e^{-a|y|+b\left|y_{3}\right|} e^{-b \frac{|y|^{2}}{4 d_{P}} \varepsilon}\left|y^{\prime}\right|^{(c-a)_{+}} \mathrm{d} y \leq \int_{\mathbb{R}} e^{-(a-b)\left|y_{3}\right|} \mathrm{d} y_{3} \int_{\mathbb{R}^{2}} e^{-b \frac{\left|y^{\prime}\right|^{2}}{4 d_{P}} \varepsilon}\left|y^{\prime}\right|^{(c-a)_{+}} \mathrm{d} y^{\prime} \\
\leq\left(\frac{d_{P}}{\varepsilon}\right)^{\frac{(c-a)_{+}}{2}+1} \int_{\mathbb{R}^{3}} e^{-(a-b)\left|y_{3}\right|} \mathrm{d} y_{3} \int_{\mathbb{R}^{2}} e^{-b \frac{\left|y^{\prime}\right|^{2}}{4}\left|y^{\prime}\right|^{(c-a)_{+}} \mathrm{d} y^{\prime} .}
\end{gathered}
$$


Now assume $a=b$ :

$$
\begin{aligned}
\int_{\mathbb{R}^{3}} e^{-a|y|+a\left|y_{3}\right|} e^{-a \mid \frac{|y|^{2}}{4 d_{P}} \varepsilon}\left|y^{\prime}\right|^{(c-a)_{+}} \mathrm{d} y & \leq \int_{\mathbb{R}^{3}} e^{-a \frac{|y|^{2}}{4 d_{P}} \varepsilon}\left|y^{\prime}\right|^{(c-a)_{+}} \mathrm{d} y \\
& =\left(\frac{d_{P}}{\varepsilon}\right)^{\frac{(c-a)_{+}+3}{2}} \int_{\mathbb{R}^{3}} e^{-a \frac{|y|^{2}}{4}\left|y^{\prime}\right|^{(c-a)_{+}} \mathrm{d} y .}
\end{aligned}
$$

Finally, if $b>a$, then by (3.3)

$$
\int_{\Omega} w_{\varepsilon, P}^{a} w_{\varepsilon, P^{*}}^{b} \frac{\left|y^{\prime}\right|^{c}}{\varepsilon^{c}} \mathrm{~d} x \leq\left(\frac{\varepsilon}{d_{P}}\right)^{b-a} e^{-(b-a) \frac{d_{P}}{\varepsilon}} \int_{\Omega} w_{\varepsilon, P}^{a} w_{\varepsilon, P^{*}}^{a} \frac{\left|y^{\prime}\right|^{c}}{\varepsilon^{c}} \mathrm{~d} x
$$

and the thesis follows from the previous cases.

Lemma 4.2. The following limit holds

$\int_{\mathbb{R}^{3}} f(w) w\left(y+\rho \ell_{3}\right) \mathrm{d} y=\frac{B}{\rho}(1+\mathrm{o}(1)) e^{-\rho}$ as $\rho \rightarrow+\infty, \quad B=A \int_{\mathbb{R}^{3}} f(w) e^{-y_{3}} \mathrm{~d} y$.

Proof. The proof is an easy consequence of Lebesgue's dominated convergence theorem. According to (1.4) for every $y \in \mathbb{R}^{3}$ we have

$$
\lim _{\rho \rightarrow+\infty} \frac{w\left(y+\rho \ell_{3}\right)}{\frac{A}{\rho} e^{-\rho}}-e^{-y_{3}}=\lim _{\rho \rightarrow+\infty} e^{-\left|y+\rho \ell_{3}\right|+\rho}-e^{-y_{3}}=0 .
$$

Observe that, if $|y| \leq \frac{\rho}{2}$, then $\left|y+\rho \ell_{3}\right| \geq \frac{\rho}{2}$; hence, by using (1.4) we get

$$
f(w) \frac{w\left(y+\rho \ell_{3}\right)}{w(\rho)} \leq 2 f(w) \frac{\rho}{\left|y+\rho \ell_{3}\right|} e^{|y|} \leq 4 f(w) e^{|y|} .
$$

On the other hand, for $|y| \geq \frac{\rho}{2}$, by (1.4) and (1.5) we obtain

$$
f(w) \frac{w\left(y+\rho \ell_{3}\right)}{w(\rho)} \leq C\|w\|_{\infty} \frac{\rho}{|y|^{3}} e^{-3|y|+\rho} \leq C\|w\|_{\infty} e^{-|y|} .
$$

Since $f(w) e^{|y|} \in L^{1}\left(\mathbb{R}^{3}\right)$, the convergence (4.1) is dominated.

Now we are ready to provide in the next two propositions the asymptotic formula for the energy $J_{\varepsilon}\left[v_{\varepsilon}\right]$.

Proposition 4.3. The following asymptotic expansion holds:

$$
\begin{aligned}
& \frac{1}{2} \int_{\Omega}\left(\varepsilon^{2}\left|\nabla \tilde{w}_{\varepsilon, P}\right|^{2}+\tilde{w}_{\varepsilon, P}^{2}\right) \mathrm{d} x-\int_{\Omega} F\left(\tilde{w}_{\varepsilon, P}\right) \mathrm{d} x \\
& \quad=I[w] \varepsilon^{3}+\varepsilon^{3} \alpha\left(\frac{d_{P}}{\varepsilon}\right)+\mathrm{O}\left(\varepsilon^{4} \sqrt{\frac{d_{P}}{\varepsilon}} e^{-2 \frac{d_{P}}{\varepsilon}}\right)+\mathrm{o}\left(\varepsilon^{6}+\varepsilon^{5} e^{-\frac{d_{P}}{\varepsilon}}+\varepsilon^{3} e^{-3 \frac{d_{P}}{\varepsilon}}\right),
\end{aligned}
$$


as $\frac{d_{P}}{\varepsilon} \rightarrow+\infty$ uniformly for $P \in \Omega$, where $\alpha: \mathbb{R}^{+} \rightarrow \mathbb{R}$ is defined by

$$
\alpha(\rho)=\frac{1}{2} \int_{\mathbb{R}^{3}} f(w) w\left(y+2 \rho \ell_{3}\right) \mathrm{d} y=(1+\mathrm{o}(1)) \frac{B}{4 \rho} e^{-2 \rho} \text { as } \rho \rightarrow+\infty .
$$

Proof. We begin by observing that by assumption (f1) and (3.2) we get

$$
F\left(\tilde{w}_{\varepsilon, P}\right)=F\left(w_{\varepsilon, P}\right)+f\left(w_{\varepsilon, P}\right)\left(\tilde{w}_{\varepsilon, P}-w_{\varepsilon, P}\right)+\mathrm{O}\left(w_{\varepsilon, P}\left(\tilde{w}_{\varepsilon, P}-w_{\varepsilon, P}\right)^{2}\right)
$$

uniformly for $x, P \in \Omega$, by which, using equation (3.1), it is easy to check that

$$
\begin{aligned}
I_{\varepsilon}\left[\tilde{w}_{\varepsilon, P}\right]:= & \frac{1}{2} \int_{\Omega}\left(\varepsilon^{2}\left|\nabla \tilde{w}_{\varepsilon, P}\right|^{2}+\tilde{w}_{\varepsilon, P}^{2}\right) \mathrm{d} x-\int_{\Omega} F\left(\tilde{w}_{\varepsilon, P}\right) \mathrm{d} x \\
= & \frac{1}{2} \int_{\Omega} f\left(w_{\varepsilon, P}\right) \tilde{w}_{\varepsilon, P} \mathrm{~d} x-\int_{\Omega} F\left(\tilde{w}_{\varepsilon, P}\right) \mathrm{d} x \\
= & -\int_{\Omega} F\left(w_{\varepsilon, P}\right) \mathrm{d} x-\frac{1}{2} \int_{\Omega} f\left(w_{\varepsilon, P}\right) \tilde{w}_{\varepsilon, P} \mathrm{~d} x+\int_{\Omega} f\left(w_{\varepsilon, P}\right) w_{\varepsilon, P} \mathrm{~d} x \\
& +\mathrm{O}\left(\int_{\Omega} w_{\varepsilon, P}\left(\tilde{w}_{\varepsilon, P}-w_{\varepsilon, P}\right)^{2} \mathrm{~d} x\right)
\end{aligned}
$$

uniformly for $P \in \Omega$. Notice that by (1.5) we have $f(w) \leq C w^{3}, F(w) \leq C w^{4}$; then (3.4) implies

$$
\begin{aligned}
& \frac{1}{2} \int_{\frac{\Omega}{\varepsilon}} f\left(w_{\varepsilon, P}(\varepsilon x)\right) w_{\varepsilon, P}(\varepsilon x) \mathrm{d} x-\int_{\frac{\Omega}{\varepsilon}} F\left(w_{\varepsilon, P}(\varepsilon x)\right) \mathrm{d} x \\
& \quad=\int_{\mathbb{R}^{3}}\left(\frac{1}{2} f(w) w-F(w)\right) \mathrm{d} x+\mathrm{o}\left(\varepsilon^{-3 \frac{d_{P}}{\varepsilon}}\right) \int_{\mathbb{R}^{3}} w \mathrm{~d} x=I[w]+\mathrm{o}\left(\varepsilon^{-3 \frac{d_{P}}{\varepsilon}}\right)
\end{aligned}
$$

as $\frac{d_{P}}{\varepsilon} \rightarrow+\infty$ uniformly for $P \in \Omega$. Hence we arrive at

$$
\begin{aligned}
I_{\varepsilon}\left[\tilde{w}_{\varepsilon, P}\right]= & I[w] \varepsilon^{3}+\frac{1}{2} \int_{\Omega} f\left(w_{\varepsilon, P}\right)\left(w_{\varepsilon, P}-\tilde{w}_{\varepsilon, P}\right) \mathrm{d} x \\
& +\mathrm{O}\left(\int_{\Omega} w_{\varepsilon, P}\left(\tilde{w}_{\varepsilon, P}-w_{\varepsilon, P}\right)^{2} \mathrm{~d} x\right)+\mathrm{o}\left(\varepsilon^{3} e^{-3 \frac{d_{P}}{\varepsilon}}\right)
\end{aligned}
$$

as $\frac{d_{P}}{\varepsilon} \rightarrow+\infty$ uniformly for $P \in \Omega$.

Next we insert the expansion provided by Proposition 3.2 in (4.4) and distinguish the two cases. First assume $P \in \Omega \backslash \Gamma_{\mu}$. Then by Part i)

$$
I_{\varepsilon}\left[\tilde{w}_{\varepsilon, P}\right]=I[w] \varepsilon^{3}+\mathrm{o}\left(\varepsilon^{6}+\varepsilon^{3} e^{-3 \frac{d_{P}}{\varepsilon}}\right)
$$


and (4.2) holds uniformly for $P \in \Omega \backslash \Gamma_{\mu}$. Next assume $P \in \Gamma_{\mu}$. Then insert the estimate provided by Part ii) of Proposition 3.2 to obtain

$$
\begin{aligned}
I_{\varepsilon}\left[\tilde{w}_{\varepsilon, P}\right]= & I[w] \varepsilon^{3}+\frac{1}{2} \int_{\Omega} f\left(w_{\varepsilon, P}\right) w_{\varepsilon, P} \mathrm{~d} x-\frac{1}{2} \int_{\Omega} f\left(w_{\varepsilon, P}\right) k_{\varepsilon, P} \mathrm{~d} x \\
& +\mathrm{O}(\varepsilon) \int_{\Omega} f\left(w_{\varepsilon, P}\right) h_{\varepsilon, P} \mathrm{~d} x+\mathrm{O}\left(\int_{\Omega} w_{\varepsilon, P} w_{\varepsilon, P^{*}}^{2} \mathrm{~d} x\right) \\
& +\mathrm{O}\left(\varepsilon^{2}\right) \int_{\Omega} h_{\varepsilon, P}^{2} \mathrm{~d} x+\mathrm{O}\left(\int_{\Omega} k_{\varepsilon, P}^{2} \mathrm{~d} x\right)+\mathrm{o}\left(\varepsilon^{3} e^{-3 \frac{d_{P}}{\varepsilon}}\right) \\
= & I[w] \varepsilon^{3}+\frac{\varepsilon^{3}}{2} \int_{\frac{\Omega}{\varepsilon}} f\left(w_{\varepsilon, P}(\varepsilon x)\right) w_{\varepsilon, P^{*}}(\varepsilon x) \mathrm{d} x-\frac{1}{2} \int_{\Omega} f\left(w_{\varepsilon, P}\right) k_{\varepsilon, P} \mathrm{~d} x \\
& +\mathrm{O}(\varepsilon) \int_{\Omega} f\left(w_{\varepsilon, P}\right) h_{\varepsilon, P} \mathrm{~d} x+o\left(\varepsilon^{3} e^{-3 \frac{d_{P}}{\varepsilon}}+\varepsilon^{5} e^{-\frac{d_{P}}{\varepsilon}}+\varepsilon^{6}\right)
\end{aligned}
$$

as $\frac{d_{P}}{\varepsilon} \rightarrow+\infty$ uniformly for $P \in \Gamma_{\mu}$, where we have used (3.8) and Lemma 4.1. Furthermore

$$
\begin{aligned}
\int_{\frac{\Omega}{\varepsilon}} f\left(w_{\varepsilon, P}(\varepsilon x)\right) w_{\varepsilon, P^{*}}(\varepsilon x) \mathrm{d} x & =\int_{\mathbb{R}^{3}} f\left(w_{\varepsilon, P}(\varepsilon x)\right) w_{\varepsilon, P^{*}}(\varepsilon x) \mathrm{d} x+\mathrm{o}\left(\varepsilon^{-3 \frac{d_{P}}{\varepsilon}}\right) \\
& =\int_{\mathbb{R}^{3}} f(w) w\left(y+\frac{2 d_{P}}{\varepsilon} \ell_{3}\right) \mathrm{d} y+\mathrm{o}\left(\varepsilon^{-3 \frac{d_{P}}{\varepsilon}}\right) \\
& =2 \alpha\left(\frac{d_{P}}{\varepsilon}\right)+\mathrm{o}\left(\varepsilon^{-3 \frac{d_{P}}{\varepsilon}}\right)
\end{aligned}
$$

as $\frac{d_{P}}{\varepsilon} \rightarrow+\infty$ uniformly for $P \in \Gamma_{\mu}$. The asymptotic formula (4.3) follows from Lemma 4.2.

After integration by parts, using (3.7),

$$
\begin{aligned}
\int_{\Omega} f\left(w_{\varepsilon, P}\right) k_{\varepsilon, P} \mathrm{~d} x & =\int_{\Omega}\left(-\varepsilon^{2} \Delta \tilde{w}_{\varepsilon, P}+\tilde{w}_{\varepsilon, P}\right) k_{\varepsilon, P} \mathrm{~d} x \\
& =\int_{\Omega} \tilde{w}_{\varepsilon, P} f\left(w_{\varepsilon, P^{*}}\right) \mathrm{d} x=\mathrm{o}\left(\varepsilon^{3} e^{-3 \frac{d P}{\varepsilon}}\right)
\end{aligned}
$$

as $\frac{d_{P}}{\varepsilon} \rightarrow+\infty$ uniformly for $P \in \Gamma_{\mu}$, where the last estimate follows from (3.3).

By (1.4) a direct computation shows that

$$
\begin{aligned}
& \left|\varepsilon^{2} \Delta\left(w_{\varepsilon, P^{*}}(x) \frac{\left|y^{\prime}\right|^{2}}{\varepsilon^{2}}\right)-w_{\varepsilon, P^{*}}(x) \frac{\left|y^{\prime}\right|^{2}}{\varepsilon^{2}}\right| \\
& \quad \leq C w_{\varepsilon, P^{*}}^{3}(x) \frac{\left|y^{\prime}\right|^{2}}{\varepsilon^{2}}+C w_{\varepsilon, P^{*}}(x)\left(1+\frac{\left|y^{\prime}\right|}{\varepsilon}\right),
\end{aligned}
$$


by which, using equation (3.6) and integrating by parts,

$$
\begin{aligned}
\mid \int_{\Omega} f\left(w_{\varepsilon, P}\right) & \left(h_{\varepsilon, P}-w_{\varepsilon, P^{*}} \frac{\left|y^{\prime}\right|^{2}}{\varepsilon^{2}}-\varepsilon^{4}\right) \mathrm{d} x \mid \\
= & \left|\int_{\Omega}\left(-\varepsilon^{2} \Delta \tilde{w}_{\varepsilon, P}+\tilde{w}_{\varepsilon, P}\right)\left(h_{\varepsilon, P}-w_{\varepsilon, P} \frac{\left|y^{\prime}\right|^{2}}{\varepsilon^{2}}-\varepsilon^{4}\right) \mathrm{d} x\right| \\
\leq & C \int_{\Omega} \tilde{w}_{\varepsilon, P} w_{\varepsilon, P^{*}}^{3} \frac{\left|y^{\prime}\right|^{2}}{\varepsilon^{2}} \mathrm{~d} x+C \int_{\Omega} \tilde{w}_{\varepsilon, P} w_{\varepsilon, P^{*}}\left(1+\frac{\left|y^{\prime}\right|}{\varepsilon}\right) \mathrm{d} x \\
& +\varepsilon^{4} \int_{\Omega} \tilde{w}_{\varepsilon, P} \mathrm{~d} x \\
\leq & C \varepsilon^{3} \sqrt{\frac{d_{P}}{\varepsilon}} e^{-2 \frac{d_{P}}{\varepsilon}}+C \varepsilon^{7}
\end{aligned}
$$

by Lemma 4.1. Finally Lemma 4.1 also gives

$$
\int_{\Omega} f\left(w_{\varepsilon, P}\right) w_{\varepsilon, P^{*}} \frac{\left|y^{\prime}\right|^{2}}{\varepsilon^{2}} \mathrm{~d} x+\varepsilon^{4} \int_{\Omega} f\left(w_{\varepsilon, P}\right) \mathrm{d} x \leq C \varepsilon^{3} e^{-2 \frac{d_{P}}{\varepsilon}}+C \varepsilon^{7} .
$$

The conclusion follows by inserting (4.6), (4.7), (4.8), (4.9) into (4.5).

Proposition 4.4. The following asymptotic expansion holds:

$$
\begin{aligned}
& \frac{\gamma}{4} \int_{\Omega} \tilde{w}_{\varepsilon, P}^{2}(-\Delta)^{-1}\left[\tilde{w}_{\varepsilon, P}^{2}\right] \mathrm{d} x \\
& =I_{2} \varepsilon^{5}-I_{3} \frac{\varepsilon^{6}}{d_{P}}+\mathrm{o}\left(\varepsilon^{11 / 2} e^{-\frac{d_{P}}{2 \varepsilon}}+\varepsilon^{5} e^{-\frac{d_{P}}{\varepsilon}}+\varepsilon^{4} e^{-2 \frac{d_{P}}{\varepsilon}}\right)+\mathrm{O}\left(\varepsilon^{6}\right),
\end{aligned}
$$

as $\frac{d_{P}}{\varepsilon} \rightarrow+\infty$ uniformly for $P \in \Omega$, where $I_{2}, I_{3}$ are positive constants; furthermore

$$
\begin{aligned}
\frac{\gamma}{4} \int_{\Omega} \tilde{w}_{\varepsilon, P}^{2}(-\Delta)^{-1}\left[\tilde{w}_{\varepsilon, P}^{2}\right] \mathrm{d} x & =I_{2} \varepsilon^{5}-I_{3} \frac{\varepsilon^{6}}{d_{P}}-I_{4} \mathcal{H}\left(\Sigma_{P}\right) \varepsilon^{6} \\
& +\mathrm{o}\left(\varepsilon^{11 / 2} e^{-\frac{d_{P}}{2 \varepsilon}}+\varepsilon^{5} e^{-\frac{d_{P}}{\varepsilon}}+\varepsilon^{4} e^{-2 \frac{d_{P}}{\varepsilon}}+\varepsilon^{6}\right)
\end{aligned}
$$

as $\frac{d_{P}}{\varepsilon} \rightarrow+\infty$ and $d_{P} \rightarrow 0$ uniformly for $P \in \Gamma_{\mu}$, where $I_{4}$ is a positive constant.

Proof. By (3.2) and a) of Proposition 2.1 we can write

$$
\begin{aligned}
\int_{\Omega} \tilde{w}_{\varepsilon, P}^{2}(-\Delta)^{-1}\left[\tilde{w}_{\varepsilon, P}^{2}\right] \mathrm{d} x= & \int_{\Omega} w_{\varepsilon, P}^{2}(-\Delta)^{-1}\left[w_{\varepsilon, P}^{2}\right] \mathrm{d} x \\
& +\mathrm{O}\left(\int_{\Omega} w_{\varepsilon, P}^{2}(-\Delta)^{-1}\left[w_{\varepsilon, P}\left(w_{\varepsilon, P}-\tilde{w}_{\varepsilon, P}\right)\right] \mathrm{d} x\right) .
\end{aligned}
$$


First assume $P \in \Omega \backslash \Gamma_{\mu}$ : b) of Proposition 2.1 and Part i) of Proposition 3.2 imply

$$
\begin{aligned}
\int_{\Omega} \tilde{w}_{\varepsilon, P}^{2}(-\Delta)^{-1}\left[\tilde{w}_{\varepsilon, P}^{2}\right] \mathrm{d} x & =\int_{\Omega} w_{\varepsilon, P}^{2}(-\Delta)^{-1}\left[w_{\varepsilon, P}^{2}\right] \mathrm{d} x+\mathrm{O}\left(\varepsilon^{4} \int_{\Omega} w_{\varepsilon, P}^{2} \mathrm{~d} x\right) \\
& =\int_{\Omega} w_{\varepsilon, P}^{2}(-\Delta)^{-1}\left[w_{\varepsilon, P}^{2}\right] \mathrm{d} x+\mathrm{O}\left(\varepsilon^{7}\right)
\end{aligned}
$$

uniformly for $P \in \Omega \backslash \Gamma_{\mu}$. Now assume $P \in \Gamma_{\mu}$; according to Part ii) of Proposition 3.2,

$$
\begin{aligned}
& \int_{\Omega} \tilde{w}_{\varepsilon, P}^{2}(-\Delta)^{-1}\left[\tilde{w}_{\varepsilon, P}^{2}\right] \mathrm{d} x=\int_{\Omega} w_{\varepsilon, P}^{2}(-\Delta)^{-1}\left[w_{\varepsilon, P}^{2}\right] \mathrm{d} x \\
& +\mathrm{O}\left(\int_{\Omega} w_{\varepsilon, P}^{2}(-\Delta)^{-1}\left[w_{\varepsilon, P} w_{\varepsilon, P^{*}}\right] \mathrm{d} x\right) \\
& +\mathrm{O}\left(\varepsilon \int_{\Omega} w_{\varepsilon, P}^{2}(-\Delta)^{-1}\left[h_{\varepsilon, P}\right] \mathrm{d} x\right) \\
& +\mathrm{O}\left(\int_{\Omega} w_{\varepsilon, P}^{2}(-\Delta)^{-1}\left[k_{\varepsilon, P}\right] \mathrm{d} x\right)
\end{aligned}
$$

uniformly for $P \in \Gamma_{\mu}$. Let us analyze the error terms: by using b) of Proposition 2.1 and Lemma 4.1 we obtain

$$
\int_{\Omega} w_{\varepsilon, P}^{2}(-\Delta)^{-1}\left[w_{\varepsilon, P} w_{\varepsilon, P^{*}}\right] \mathrm{d} x \leq C \varepsilon^{3 / 2} e^{-2 \frac{d_{P}}{\varepsilon}} \int_{\Omega} w_{\varepsilon, P}^{2} \mathrm{~d} x \leq C \varepsilon^{9 / 2} e^{-2 \frac{d_{P}}{\varepsilon}}
$$

uniformly for $P \in \Gamma_{\mu}$. Again by b) of Proposition 2.1 and (3.8) we get

$$
\begin{aligned}
\int_{\Omega} w_{\varepsilon, P}^{2}(-\Delta)^{-1}\left[h_{\varepsilon, P}\right] \mathrm{d} x & \leq C \varepsilon^{3 / 2}\left(\varepsilon^{2}+e^{-\frac{2 d_{P}}{3 \varepsilon}}\right) \int_{\mathbb{R}^{3}} w_{\varepsilon, P}^{2} \mathrm{~d} x \\
& \leq C\left(\varepsilon^{13 / 2}+\varepsilon^{9 / 2} e^{-\frac{2 d_{P}}{3 \varepsilon}}\right) .
\end{aligned}
$$

and

$$
\int_{\Omega} w_{\varepsilon, P}^{2}(-\Delta)^{-1}\left[k_{\varepsilon, P}\right] \mathrm{d} x \leq C \varepsilon^{3 / 2} e^{-2 \frac{d_{P}}{\varepsilon}} \int_{\mathbb{R}^{3}} w_{\varepsilon, P}^{2} \mathrm{~d} x \leq C \varepsilon^{9 / 2} e^{-2 \frac{d_{P}}{\varepsilon}}
$$

uniformly for $P \in \Gamma_{\mu}$. Combining (4.12), (4.13), (4.14), (4.15) and (4.16) we arrive at

$\int_{\Omega} \tilde{w}_{\varepsilon, P}^{2}(-\Delta)^{-1}\left[\tilde{w}_{\varepsilon, P}^{2}\right] \mathrm{d} x=\int_{\Omega} w_{\varepsilon, P}^{2}(-\Delta)^{-1}\left[w_{\varepsilon, P}^{2}\right] \mathrm{d} x+\mathrm{o}\left(\varepsilon^{11 / 2} e^{-\frac{d_{P}}{2 \varepsilon}}+\varepsilon^{4} e^{-2 \frac{d_{P}}{\varepsilon}}+\varepsilon^{6}\right)$

as $\frac{d_{P}}{\varepsilon} \rightarrow+\infty$ uniformly for $P \in \Omega$. Thus it is sufficient to estimate

$$
\int_{\Omega} w_{\varepsilon, P}^{2}(-\Delta)^{-1}\left[w_{\varepsilon, P}^{2}\right] \mathrm{d} x
$$


To this aim denote by $V_{0}$ the unique solution of

$$
\Delta V_{0}+w^{2}=0 \text { in } \mathbb{R}^{3}, \quad V_{0} \rightarrow 0 \text { as }|x| \rightarrow+\infty
$$

i.e. $V_{0}(x)=\frac{1}{4 \pi} \int_{\mathbb{R}^{3}} \frac{1}{|x-z|} w^{2}(z) \mathrm{d} z$. Then $V_{0}$ is radial and its equation in radial coordinates becomes $\left(r^{2} V_{0}^{\prime}\right)^{\prime}+r^{2} w^{2}=0$ by which, after integration,

$$
\begin{aligned}
V_{0}^{\prime}(r) & =-\frac{1}{r^{2}} \int_{0}^{r} s^{2} w^{2} \mathrm{~d} s \\
& =-\frac{1}{r^{2}}\left(\int_{0}^{+\infty} s^{2} w^{2} \mathrm{~d} s+\mathrm{O}\left(e^{-2 r}\right)\right)=-\frac{c_{0}}{r^{2}}\left(1+\mathrm{O}\left(e^{-2 r}\right)\right)
\end{aligned}
$$

for some $c_{0}>0$ and, by integrating again,

$$
V_{0}(r)=-\int_{r}^{+\infty} V_{0}^{\prime}(s) \mathrm{d} s=\frac{c_{0}}{r}+\mathrm{O}\left(e^{-2 r}\right) .
$$

Now set

$$
V_{\varepsilon, P}(x)=V_{0}\left(\frac{x-P}{\varepsilon}\right), \quad x \in \Omega, P \in \mathbb{R}^{3} .
$$

First assume $P \in \Omega \backslash \Gamma_{\mu}$. Note that $(-\Delta)^{-1}\left[w_{\varepsilon, P}^{2}\right]-\varepsilon^{2} V_{\varepsilon, P}$ solve

$\Delta\left((-\Delta)^{-1}\left[w_{\varepsilon, P}^{2}\right]-\varepsilon^{2} V_{\varepsilon, P}\right)=0$ in $\Omega, \quad(-\Delta)^{-1}\left[w_{\varepsilon, P}^{2}\right]-\varepsilon^{2} V_{\varepsilon, P}=-\varepsilon^{2} V_{\varepsilon, P}$ on $\partial \Omega$ and by (4.18) $V_{\varepsilon, P}=\mathrm{O}(\varepsilon)$ uniformly for $x \in \partial \Omega$ and $P \in \Omega \backslash \Gamma_{\mu}$. From the maximum principle it follows that $(-\Delta)^{-1}\left[w_{\varepsilon, P}^{2}\right]-\varepsilon^{2} V_{\varepsilon, P}=\mathrm{O}\left(\varepsilon^{3}\right)$ uniformly for $x \in \partial \Omega$ and $P \in \Omega \backslash \Gamma_{\mu}$. Hence using (3.4) we obtain

$$
\begin{aligned}
\int_{\Omega} w_{\varepsilon, P}^{2}(-\Delta)^{-1}\left[w_{\varepsilon, P}^{2}\right] & =\varepsilon^{2} \int_{\Omega} w_{\varepsilon, P}^{2} V_{\varepsilon, P} \mathrm{~d} x+\mathrm{O}\left(\varepsilon^{3}\right) \int_{\Omega} w_{\varepsilon, P}^{2} \\
& =\varepsilon^{5} \int_{\mathbb{R}^{3}} w^{2} V_{0} \mathrm{~d} x+\mathrm{o}\left(\varepsilon^{5} e^{-\frac{d P}{\varepsilon}}\right)+\mathrm{O}\left(\varepsilon^{6}\right)
\end{aligned}
$$

and (4.10) holds uniformly for $P \in \Omega \backslash \Gamma_{\mu}$. Now we assume $P \in \Gamma_{\mu}$ and decompose

$$
(-\Delta)^{-1}\left[w_{\varepsilon, P}^{2}\right]=\varepsilon^{2}\left(V_{\varepsilon, P}-V_{\varepsilon, P *}+Z_{\varepsilon, P}-W_{\varepsilon, P}\right)
$$

where $Z_{\varepsilon, P}$ and $W_{\varepsilon, P}$ solve the following problems:

$$
\begin{gathered}
\Delta Z_{\varepsilon, P}+\frac{1}{\varepsilon^{2}} w_{\varepsilon, P^{*}}^{2}=0 \text { in } \Omega, \quad Z_{\varepsilon, P}=0 \text { on } \partial \Omega, \\
\Delta W_{\varepsilon, P}=0 \text { in } \Omega, \quad W_{\varepsilon, P}=V_{\varepsilon, P}-V_{\varepsilon, P^{*}} \text { on } \partial \Omega .
\end{gathered}
$$

For the sake of clarity we divide the remaining part of the proof into 4 steps. In order to simplify the notation, during the steps we will write $d$ and $\mathcal{H}_{j}$ in the 
place of $d_{P}$ and $\mathcal{H}_{j}\left(\Sigma_{P}\right)$, since this can be done without causing confusion. We let it be understood that all the asymptotic estimates hold uniformly with respect to the choice of $P \in \Gamma_{\mu}$.

Step 1. The following holds:

$$
V_{\varepsilon, P}(x)-V_{\varepsilon, P^{*}}(x)=c_{0} \varepsilon \frac{\sum_{j=1,2} \mathcal{H}_{j} \hat{y}_{j}^{\prime 2}}{\left(1+|\hat{y}|^{2}\right)^{3 / 2}}+\mathrm{o}(\varepsilon) \text { as } \frac{d}{\varepsilon} \rightarrow+\infty, d \rightarrow 0^{+},
$$

uniformly for $x \in \partial \Omega$, where $\hat{y}=\frac{y}{d}=\frac{T_{P} x}{d}$.

By (3.5), we compute

$|x-P|=\sqrt{d^{2}+|y|^{2}-2 d y_{3}}=\sqrt{d^{2}+|y|^{2}+\mathrm{O}\left(d\left|y^{\prime}\right|^{2}\right)}=d \sqrt{1+|\hat{y}|^{2}+\mathrm{o}\left(\left|\hat{y}^{\prime}\right|^{2}\right)}$ as $d \rightarrow 0^{+}$uniformly for $x \in \partial \Omega$. In the same way

$$
\begin{aligned}
\left|x-P^{*}\right| & =\sqrt{d^{2}+|y|^{2}+2 d y_{3}}=d \sqrt{1+|\hat{y}|^{2}+\mathrm{o}\left(\left|\hat{y}^{\prime}\right|^{2}\right)}, \\
r_{x, P} & =d\left(\sqrt{1+|\hat{y}|^{2}+\mathrm{o}\left(\left|\hat{y}^{\prime}\right|^{2}\right)}\right.
\end{aligned}
$$

as $d \rightarrow 0^{+}$uniformly for $x \in \partial \Omega$ and $r_{x, P} \in \mathcal{I}_{x, P}:=(\min \{|x-P|, \mid x-$ $\left.\left.P^{*} \mid\right\}, \max \left\{|x-P|,\left|x-P^{*}\right|\right\}\right)$. Hence by using again (3.5) we get

$$
\begin{aligned}
\frac{\left|x-P^{*}\right|-|x-P|}{r_{x, P}^{2}} & =\frac{\left|x-P^{*}\right|^{2}-|x-P|^{2}}{r_{x, P}^{2}\left(|x-P|+\left|x-P^{*}\right|\right)} \\
& =\frac{2 \mathrm{~d} y_{3}}{d^{3}\left(1+|\hat{y}|^{2}+\mathrm{o}\left(\left|\hat{y}^{\prime}\right|^{2}\right)\right)^{3 / 2}} \\
& =\frac{d^{3} \sum_{j} \mathcal{H}_{j} \hat{y}_{j}^{\prime 2}+2 d \omega_{P}\left(d \hat{y}^{\prime}\right)}{d^{3}\left(1+|\hat{y}|^{2}+\mathrm{o}\left(\left|\hat{y}^{\prime}\right|^{2}\right)\right)^{3 / 2}} \\
& =\frac{\sum_{j} \mathcal{H}_{j} \hat{y}_{j}^{\prime 2}}{\left(1+|\hat{y}|^{2}+\mathrm{o}\left(\left|\hat{y}^{\prime}\right|^{2}\right)\right)^{3 / 2}}+\frac{\mathrm{O}\left(\omega_{P}\left(d \hat{y}^{\prime}\right)\right)}{d^{2}\left(1+|\hat{y}|^{2}\right)^{3 / 2}} \\
& =\frac{\sum_{j} \mathcal{H}_{j} \hat{y}_{j}^{\prime 2}}{\left(1+|\hat{y}|^{2}\right)^{3 / 2}}+\mathrm{o}(1)+\frac{\mathrm{O}\left(\omega_{P}\left(d \hat{y}^{\prime}\right)\right)}{d^{2}\left(1+|\hat{y}|^{2}\right)^{3 / 2}}
\end{aligned}
$$

as $d \rightarrow 0$ uniformly for $x \in \partial \Omega$ and $r_{x, P} \in \mathcal{I}_{x, P}$. Observe that

$$
\frac{\omega_{P}\left(d \hat{y}^{\prime}\right)}{d^{2}\left(1+|\hat{y}|^{2}\right)^{3 / 2}}=\mathrm{o}(1) \text { as } d \rightarrow 0 \text { uniformly for } x \in \partial \Omega .
$$


Indeed by (3.5)

$$
\frac{\omega_{P}\left(d \hat{y}^{\prime}\right)}{d^{2}\left(1+|\hat{y}|^{2}\right)^{3 / 2}}=\frac{\mathrm{o}\left(d^{2}\left|\hat{y}^{\prime}\right|^{2}\right)}{d^{2}\left(1+|\hat{y}|^{2}\right)^{3 / 2}}=\mathrm{o}(1) \text { uniformly for }\left|\hat{y}^{\prime}\right| \leq \frac{1}{\sqrt{d}} .
$$

While, if $\left|\hat{y}^{\prime}\right| \geq \frac{1}{\sqrt{d}}$, then $\frac{\omega_{P}\left(d \hat{y}^{\prime}\right)}{d^{2}\left(1+|\hat{y}|^{2}\right)^{3 / 2}} \leq \frac{C\left|y^{\prime}\right|^{2}}{\left(1+|\hat{y}|^{2}\right)^{3 / 2}}=\mathrm{o}(1)$. By inserting (4.23) in (4.22) we deduce

$$
\frac{\left|x-P^{*}\right|-|x-P|}{r_{x, P}^{2}}=\sum_{j} \mathcal{H}_{j} \frac{\hat{y}_{j}^{\prime 2}}{\left(1+|\hat{y}|^{2}\right)^{3 / 2}}+\mathrm{o}(1) \text { as } d \rightarrow 0^{+}
$$

uniformly for $x \in \partial \Omega$ and $r_{x, P} \in \mathcal{I}_{x, P}$.

Then by the mean value theorem, using (4.17), we derive

$$
\begin{aligned}
V_{\varepsilon, P}(x)-V_{\varepsilon, P^{*}}(x) & =-c_{0} \varepsilon\left(1+\mathrm{O}\left(e^{-2 \frac{d}{\varepsilon}}\right)\right) \frac{|x-P|-\left|x-P^{*}\right|}{r_{x, P}^{2}} \\
& =c_{0} \varepsilon\left(1+\mathrm{O}\left(e^{-2 \frac{d}{\varepsilon}}\right)\right) \frac{\sum_{j} \mathcal{H}_{j} \hat{y}_{j}^{\prime 2}}{\left(1+|\hat{y}|^{2}\right)^{3 / 2}}+\mathrm{o}(\varepsilon) \\
& =\frac{c_{0} \varepsilon \sum_{j} \mathcal{H}_{j} \hat{y}_{j}^{\prime 2}}{\left(1+|\hat{y}|^{2}\right)^{3 / 2}}+\mathrm{o}(\varepsilon)
\end{aligned}
$$

as $d \rightarrow 0^{+}, \frac{d}{\varepsilon} \rightarrow+\infty$ uniformly for $x \in \partial \Omega$. Hence Step 1 holds.

Step 2. For $P \in \Gamma_{\mu}$ such that $\frac{d}{\varepsilon}$ is sufficiently large we have

$$
V_{\varepsilon, P}(x)-V_{\varepsilon, P^{*}}(x)=\mathrm{O}(\varepsilon) \text { uniformly for } x \in \partial \Omega .
$$

By (4.18), fixed $\eta>0$, for $P \in \Gamma_{\mu}$ with $d \geq \eta$ we have

$$
V_{\varepsilon, P}(x), V_{\varepsilon, P *}(x) \leq \frac{c_{0} \varepsilon}{\eta}+\mathrm{O}\left(e^{-\frac{2 \eta}{\varepsilon}}\right)=\mathrm{O}(\varepsilon) \text { uniformly for } x \in \partial \Omega .
$$

Then Step 2 follows by Step 1 .

Step 3. Set $\hat{W}_{\varepsilon, P}(\hat{y})=W_{\varepsilon, P}(x)$ where $\hat{y}=\frac{T_{P} x}{d}$. Then for $P \in \Gamma_{\mu}$ such that $\frac{d}{\varepsilon}$ is sufficiently large

$$
\hat{W}_{\varepsilon, P}(\hat{y})=\mathrm{O}(\varepsilon) \text { uniformly for } \hat{y} \in \frac{T_{P} \Omega}{d} .
$$

Furthermore $\frac{1}{\varepsilon} \hat{W}_{\varepsilon, P} \rightarrow \hat{W}_{1}+\hat{W}_{2}$ as $\frac{d}{\varepsilon} \rightarrow+\infty$ and $d \rightarrow 0^{+}$uniformly on compact sets of $\mathbb{R}_{+}^{3}$, where $\hat{W}_{j}$ satisfies

$$
\Delta \hat{W}_{j}=0 \text { in } \mathbb{R}_{+}^{3}, \quad \hat{W}_{j}=c_{0} \mathcal{H}_{j} \frac{\hat{y}_{j}^{\prime 2}}{\left(1+\left|\hat{y}^{\prime}\right|^{2}\right)^{3 / 2}} \text { on } \partial \mathbb{R}_{+}^{3} .
$$


By Step 2, from the comparison principle it follows immediately that if $\frac{d}{\varepsilon}$ is large enough $\hat{W}_{\varepsilon, P}(\hat{y})=\mathrm{O}(\varepsilon)$ uniformly for $\hat{y} \in \frac{T_{P} \Omega}{d}$.

By (4.21) and Step 1, from the comparison principle it follows that as $d \rightarrow 0^{+}$ and $\frac{d}{\varepsilon} \rightarrow+\infty$

$$
\hat{W}_{\varepsilon, P}(\hat{y})=\varepsilon \sum_{j=1,2} \hat{W}_{\varepsilon, P}^{j}(\hat{y})+\mathrm{o}(\varepsilon)
$$

uniformly for $\hat{y} \in \frac{T_{P} \Omega}{d}$, where $\hat{W}_{\varepsilon, P}^{j}$, solve

$$
\begin{aligned}
\Delta \hat{W}_{\varepsilon, P}^{j} & =0 \text { in } \frac{T_{P} \Omega}{d}, \\
\hat{W}_{\varepsilon, P}^{j} & =g_{j}:=c_{0} \mathcal{H}_{j} \frac{\hat{y}_{j}^{\prime 2}}{\left(1+|\hat{y}|^{2}\right)^{3 / 2}} \text { on } \partial\left(\frac{T_{P} \Omega}{d}\right), \quad j=1,2 .
\end{aligned}
$$

Denote by $D^{1,2}$ the closure of $C_{0}^{\infty}\left(\mathbb{R}^{3}\right)$ with respect to the norm $\|u\|_{D^{1,2}}^{2}=$ $\int_{\mathbb{R}^{3}}|\nabla u|^{2} \mathrm{~d} x$. An easy computation shows that $g_{j} \in D^{1,2}$. By multiplying both members of (4.26) by $\hat{W}_{\varepsilon, P}^{j}-g_{j}$ and integrating on $\frac{T_{P} \Omega}{d}$ we obtain

$$
\begin{aligned}
\int_{\frac{T_{P} \Omega}{d}}\left|\nabla\left(\hat{W}_{\varepsilon, P}^{j}-g_{j}\right)\right|^{2} d \hat{y} & =-\int_{\frac{T_{P} \Omega}{d}} \nabla\left(\hat{W}_{\varepsilon, P}^{j}-g_{j}\right) \nabla g_{j} d \hat{y} \\
& \leq\left\|\hat{W}_{\varepsilon, P}^{j}-g_{j}\right\|_{D^{1,2}}\left\|g_{j}\right\|_{D^{1,2}}
\end{aligned}
$$

where we have set $\hat{W}_{\varepsilon, P}^{j}=g_{j}$ in $\mathbb{R}^{3} \backslash \frac{T_{P} \Omega}{d}$. Hence we deduce that $\left\{\hat{W}_{\varepsilon, P}^{j}-g_{j}\right\}_{\varepsilon}$ and, consequently, $\left\{\hat{W}_{\varepsilon, P}^{j}\right\}_{\varepsilon}$ are bounded in $D^{1,2}$. Then, if we consider a generic sequence $\varepsilon_{n} \rightarrow 0^{+}$and $P_{n} \in \Omega$ such that $d_{P_{n}} \rightarrow 0$ and $\frac{d P_{n}}{\varepsilon_{n}} \rightarrow+\infty$, up to a subsequence we may assume $\hat{W}_{\varepsilon_{n}, P_{n}}^{j} \rightarrow \hat{W}_{j}$ as $n \rightarrow+\infty$ weakly in $D^{1,2}$ and a.e. in $\mathbb{R}^{3}$. Note that, since by construction $\chi_{\frac{1}{d P_{n}}} T_{P_{n}} \Omega \rightarrow \chi_{\mathbb{R}_{+}^{3}}$ (denoting $\chi$ the characteristic function), then $\hat{W}_{\varepsilon_{n}, P_{n}}^{j} \rightarrow g_{j}$ a.e. in $\mathbb{R}_{-}^{3}$ and $\left.\hat{W}_{j}\right|_{\mathbb{R}_{+}^{3}}$ solves (4.25). The uniqueness of the solution of (4.25) implies that all the family $\hat{W}_{\varepsilon, P}^{j}$ converges to $\hat{W}_{j}$ in $D^{1,2}$ and a.e. in $\mathbb{R}_{+}^{3}$ as $d \rightarrow 0^{+}$and $\frac{d}{\varepsilon} \rightarrow+\infty$.

For every fixed compact set $K \subset \mathbb{R}_{+}^{3}$, for small $d$ we have $K \subset \frac{T_{P} \Omega}{d}$. Since $\left\{\hat{W}_{\varepsilon, P}^{j}\right\}_{\varepsilon}$ are harmonic functions uniformly bounded on $\frac{T_{P} \Omega}{d}$, then the classical Schauder's internal estimates imply that $\hat{W}_{\varepsilon, P}^{j} \rightarrow \hat{W}_{j}$ uniformly in $K$. 
Step 4. End of the proof:

$$
\begin{aligned}
& \frac{\gamma}{4} \int_{\Omega} w_{\varepsilon, P}^{2}\left(-\Delta^{-1}\right)\left[w_{\varepsilon, P}^{2}\right] \mathrm{d} x= \varepsilon^{5}\left(I_{2}-I_{3} \frac{\varepsilon}{d}+\mathrm{O}(\varepsilon)+\mathrm{o}\left(e^{-\frac{d}{\varepsilon}}\right)\right) \text { as } \frac{d}{\varepsilon} \rightarrow+\infty, \\
& \frac{\gamma}{4} \int_{\Omega} w_{\varepsilon, P}^{2}\left(-\Delta^{-1}\right)\left[w_{\varepsilon, P}^{2}\right] \mathrm{d} x= \varepsilon^{5}\left(I_{2}-I_{3} \frac{\varepsilon}{d}-\varepsilon I_{4} \mathcal{H}\left(\Sigma_{P}\right)+\mathrm{o}(\varepsilon)+\mathrm{o}\left(e^{-\frac{d}{\varepsilon}}\right)\right) \\
& \text { as } \frac{d}{\varepsilon} \rightarrow+\infty, d \rightarrow 0^{+}
\end{aligned}
$$

From (4.19) we obtain

$$
\begin{aligned}
\int_{\Omega} w_{\varepsilon, P}^{2}\left(-\Delta^{-1}\right)\left[w_{\varepsilon, P}^{2}\right] \mathrm{d} x= & \varepsilon^{5} \int_{\frac{\Omega}{\varepsilon}} w_{\varepsilon, P}^{2}(\varepsilon x)\left(V_{\varepsilon, P}(\varepsilon x)-V_{\varepsilon, P^{*}}(\varepsilon x)\right) \mathrm{d} x \\
& +\varepsilon^{5} \int_{\frac{\Omega}{\varepsilon}} w_{\varepsilon, P}^{2}(\varepsilon x) Z_{\varepsilon, P}(\varepsilon x) \mathrm{d} x \\
& -\varepsilon^{5} \int_{\frac{\Omega}{\varepsilon}} w_{\varepsilon, P}^{2}(\varepsilon x) W_{\varepsilon, P}(\varepsilon x) \mathrm{d} x .
\end{aligned}
$$

(3.4) and (4.18) imply

$$
\begin{aligned}
& \frac{\gamma}{4} \int_{\frac{\Omega}{\varepsilon}} w_{\varepsilon, P}^{2}(\varepsilon x)\left(V_{\varepsilon, P}(\varepsilon x)-V_{\varepsilon, P^{*}}(\varepsilon x)\right) \mathrm{d} x \\
& \quad=\frac{\gamma}{4} \int_{\mathbb{R}^{3}} w^{2} V_{0} \mathrm{~d} x-\frac{\gamma}{4} \int_{\mathbb{R}^{3}} w^{2} V_{0}\left(x-\frac{P^{*}-P}{\varepsilon}\right) \mathrm{d} x+\mathrm{o}\left(\varepsilon^{-\frac{d}{\varepsilon}}\right) \\
& \quad=I_{2}-\frac{\gamma}{4} \int_{\mathbb{R}^{3}} w^{2} \frac{c_{0}}{\left|x-\frac{P-P^{*}}{\varepsilon}\right|} \mathrm{d} x+\mathrm{O}\left(\int_{\mathbb{R}^{3}} w^{2} e^{-2\left|x-\frac{P-P^{*}}{\varepsilon}\right|} \mathrm{d} x\right)+\mathrm{o}\left(\varepsilon^{-\frac{d}{\varepsilon}}\right) \\
& \quad=I_{2}-\gamma \pi c_{0} V_{0}\left(\frac{P-P^{*}}{\varepsilon}\right)+\mathrm{O}\left(e^{-\frac{2 d}{\varepsilon}} \int_{\mathbb{R}^{3}} w^{2} e^{|x|} \mathrm{d} x\right)+\mathrm{O}\left(e^{-\frac{d}{\varepsilon}}\right) \\
& \quad=I_{2}-I_{3} \frac{\varepsilon}{d}+\mathrm{o}\left(e^{-\frac{d}{\varepsilon}}\right),
\end{aligned}
$$

where we have set $I_{2}=\frac{\gamma}{4} \int_{\mathbb{R}^{3}} w^{2} V_{0}$ and $I_{3}=\frac{\gamma \pi c_{0}^{2}}{2}$.

Using c) of Proposition 2.1, Lemma 3.1 and (4.20), for $\frac{d}{\varepsilon}$ large enough we get

$$
\begin{aligned}
\int_{\frac{\Omega}{\varepsilon}} w_{\varepsilon, P}^{2}(\varepsilon x) Z_{\varepsilon, P}(\varepsilon x) \mathrm{d} x & \leq C\left\|Z_{\varepsilon, P}\right\|_{L^{\infty}(\Omega)} \\
& \leq C\left\|w_{\varepsilon, P^{*}}^{2}\right\|_{L^{\infty}(\Omega)}+C \varepsilon^{-3}\left\|w_{\varepsilon, P^{*}}^{2}\right\|_{L^{1}(\Omega)} \leq C e^{-\frac{4 d}{3 \varepsilon}},
\end{aligned}
$$


and, using (4.24),

$$
\int_{\frac{\Omega}{\varepsilon}} w_{\varepsilon, P}^{2}(\varepsilon x) W_{\varepsilon, P}(\varepsilon x) \mathrm{d} x=\mathrm{O}(\varepsilon)
$$

then the first part of the thesis of Step 4 follows. Finally as $\frac{d}{\varepsilon} \rightarrow+\infty$

$$
\begin{aligned}
\int_{\frac{\Omega}{\varepsilon}} w_{\varepsilon, P}^{2}(\varepsilon x) W_{\varepsilon, P}(\varepsilon x) \mathrm{d} x & =\int_{\left|x-\frac{P}{\varepsilon}\right| \leq \sqrt{\frac{d}{\varepsilon}}} w_{\varepsilon, P}^{2}(\varepsilon x) W_{\varepsilon, P}(\varepsilon x) \mathrm{d} z+\mathrm{o}(\varepsilon) \\
& =\int_{\left|y-\frac{d}{\varepsilon} \ell_{3}\right| \leq \sqrt{\frac{d}{\varepsilon}}} w^{2}\left(y-\frac{d}{\varepsilon} \ell_{3}\right) \hat{W}_{\varepsilon, P}\left(\frac{\varepsilon}{d} y\right) \mathrm{d} y+\mathrm{o}(\varepsilon) \\
& =\int_{|z| \leq \sqrt{\frac{d}{\varepsilon}}} w^{2}(z) \hat{W}_{\varepsilon, P}\left(\ell_{3}+\frac{\varepsilon}{d} z\right) \mathrm{d} z+\mathrm{o}(\varepsilon) .
\end{aligned}
$$

By Step 3 we immediately deduce $\frac{1}{\varepsilon} \hat{W}_{\varepsilon, P}\left(\ell_{3}+\frac{\varepsilon}{d} z\right) \rightarrow \hat{W}_{1}\left(\ell_{3}\right)+\hat{W}_{2}\left(\ell_{3}\right)$ uniformly on $|z| \leq \sqrt{\frac{d}{\varepsilon}}$ as $\frac{d}{\varepsilon} \rightarrow+\infty$ and $d \rightarrow 0^{+}$. On the other hand it is well known (see, for example [22], page 37) that the following representation formula holds for (4.25):

$$
\hat{W}_{j}(\hat{y})=c_{0} \mathcal{H}_{j} \frac{\hat{y}_{3}}{2 \pi} \int_{\mathbb{R}^{2}} \frac{\left|\hat{z}_{j}^{\prime}\right|^{2}}{\left(1+\left|\hat{z}^{\prime}\right|^{2}\right)^{3 / 2}} \frac{d \hat{z}^{\prime}}{\left[\left(\hat{z}_{1}-\hat{y}_{1}\right)^{2}+\left(\hat{z}_{2}-\hat{y}_{2}\right)^{2}+\hat{y}_{3}^{2}\right]^{3 / 2}},
$$

by which

$$
\hat{W}_{j}\left(\ell_{3}\right)=c_{0} \mathcal{H}_{j} \frac{1}{2 \pi} \int_{\mathbb{R}^{2}} \frac{\left|\hat{z}_{j}^{\prime}\right|^{2}}{\left(1+\left|\hat{z}^{\prime}\right|^{2}\right)^{3}} d \hat{z}^{\prime}, \quad j=1,2 .
$$

Hence we obtain

$$
\frac{\gamma}{4} \int_{\frac{\Omega}{\varepsilon}} w_{\varepsilon, P}^{2}(\varepsilon x) W_{\varepsilon, P}(\varepsilon x) \mathrm{d} x=\varepsilon \frac{I_{4}}{2} \sum_{j=1,2} \mathcal{H}_{j}+\mathrm{o}(\varepsilon)=\varepsilon I_{4} \mathcal{H}\left(\Sigma_{P}\right)+\mathrm{o}(\varepsilon)
$$

as $\frac{d}{\varepsilon} \rightarrow+\infty$ and $d \rightarrow 0^{+}$, where $I_{4}=\frac{c_{0} \gamma}{2} \int_{\mathbb{R}^{3}} w^{2} \mathrm{~d} x \frac{1}{2 \pi} \int_{\mathbb{R}^{2}} \frac{\left|\hat{z}_{1}^{\prime}\right|^{2}}{\left(1+\left|\hat{z}^{\prime}\right|^{2}\right)^{3}} d \hat{z}^{\prime}$.

\section{Least-energy solutions}

The object of this section is to prove the first part of Theorem 1.1; in particular we are going to show the existence of a least-energy solution $v_{\varepsilon}$ for the problem (1.6), i.e. a solution with the important property of minimizing the energy $J_{\varepsilon}$ among all solutions of (1.6). Furthermore we will provide a detailed description of its shape which has the form of a single spike near the boundary. The more delicate matter of the location of the spike at the boundary will be the subject of the next Section which concludes the proof. 
We begin with the following existence theorem.

Theorem 5.1. For every $\varepsilon>0$ the value $J_{\varepsilon}^{*}$ defined by (1.8) is a positive critical value of $J_{\varepsilon}$ with critical point $v_{\varepsilon}$. Furthermore $J_{\varepsilon}^{*}$ is the least-energy critical value of $J_{\varepsilon}$. Finally for every family $d_{\varepsilon} \rightarrow 0^{+}$with $\frac{d_{\varepsilon}}{\varepsilon} \rightarrow+\infty$

$$
\begin{aligned}
J_{\varepsilon}^{*} \leq & I[w] \varepsilon^{3}+I_{2} \varepsilon^{5}-I_{3} \frac{\varepsilon^{6}}{d_{\varepsilon}}+\varepsilon^{3} \alpha\left(\frac{d_{\varepsilon}}{\varepsilon}\right)-I_{4} \varepsilon^{6} \mathcal{H}_{0}+\mathrm{O}\left(\varepsilon^{4} \sqrt{\frac{d_{\varepsilon}}{\varepsilon}} e^{-2 \frac{d_{\varepsilon}}{\varepsilon}}\right) \\
& +\mathrm{o}\left(\varepsilon^{6}+\varepsilon^{11 / 2} e^{-\frac{d_{\varepsilon}}{2 \varepsilon}}+\varepsilon^{5} e^{-\frac{d_{\varepsilon}}{\varepsilon}}+\varepsilon^{4} e^{-2 \frac{d_{\varepsilon}}{\varepsilon}}+\varepsilon^{3} e^{-3 \frac{d_{\varepsilon}}{\varepsilon}}\right) \text { as } \varepsilon \rightarrow 0^{+}
\end{aligned}
$$

where $\alpha: \mathbb{R}^{+} \rightarrow \mathbb{R}$ has been introduced in Proposition 4.3.

Proof. Fix $\varepsilon>0$. The object is to apply the Mountain-Pass Lemma to the functional $J_{\varepsilon}$. To this aim we first prove that $J_{\varepsilon}$ satisfies the Palais-Smale condition. Let $\left\{v_{n}\right\}_{n} \subset H_{0}^{1}(\Omega)$ be such that

$$
\left|J_{\varepsilon}\left[v_{n}\right]\right| \leq M, \quad J_{\varepsilon}^{\prime}\left[v_{n}\right] \rightarrow 0
$$

for some constant $M>0$. Then, using assumption (f4),

$$
\begin{aligned}
4 M+\mathrm{o}(1)\left\|v_{n}\right\|_{H^{1}} & \geq 4 J_{\varepsilon}\left[v_{n}\right]-\left\langle J_{\varepsilon}^{\prime}\left[v_{n}\right], v_{n}\right\rangle \\
& =3 \varepsilon^{2} \int_{\Omega}\left|\nabla v_{n}\right|^{2} \mathrm{~d} x+3 \int_{\Omega}\left|v_{n}\right|^{2} \mathrm{~d} x+\int_{\Omega}\left(f\left(v_{n}\right) v_{n}-4 F\left(v_{n}\right)\right) \mathrm{d} x \\
& \geq 3 \varepsilon^{2} \int_{\Omega}\left|\nabla v_{n}\right|^{2} \mathrm{~d} x+3 \int_{\Omega}\left|v_{n}\right|^{2} \mathrm{~d} x .
\end{aligned}
$$

Hence $\left\{v_{n}\right\}_{n}$ turns out to be bounded in $H_{0}^{1}(\Omega)$; then, up to a subsequence, using Rellich's theorem, for some $v \in H_{0}^{1}(\Omega)$

$$
v_{n} \rightarrow v \text { in } H_{0}^{1}(\Omega), \quad v_{n} \rightarrow v \text { in } L^{s}(\Omega) \text { for } 1 \leq s<6 .
$$

Using the continuity of $(-\Delta)^{-1}: H^{-1}(\Omega) \rightarrow H_{0}^{1}(\Omega)$ we deduce

$$
(-\Delta)^{-1}\left[v_{n}^{2}\right] \rightarrow(-\Delta)^{-1}\left[v^{2}\right] \text { in } H_{0}^{1}(\Omega)
$$

and, consequently, using assumption (f3),

$$
-v_{n}+f\left(v_{n}\right)-\gamma(-\Delta)^{-1}\left[v_{n}^{2}\right] v_{n} \rightarrow-v+f(v)-\gamma(-\Delta)^{-1}\left[v^{2}\right] v \text { in } H^{-1}(\Omega) .
$$

Hence we arrive at

$$
\begin{aligned}
-\varepsilon^{2} \Delta v_{n}= & J_{\varepsilon}^{\prime}\left[v_{n}\right]-v_{n}+f\left(v_{n}\right)-\gamma(-\Delta)^{-1}\left[v_{n}^{2}\right] v_{n} \\
& \rightarrow-v+f(v)-\gamma(-\Delta)^{-1}\left[v^{2}\right] v \text { in } H^{-1}(\Omega) .
\end{aligned}
$$


Again the continuity of $(-\Delta)^{-1}$ allows us to conclude $\varepsilon^{2} v_{n} \rightarrow(-\Delta)^{-1}[-v+$ $\left.f(v)-\gamma(-\Delta)^{-1}\left[v^{2}\right] v\right]$ in $H_{0}^{1}(\Omega)$. Hence the P.-S. condition is satisfied for $J_{\varepsilon}$.

Next observe that $J_{\varepsilon}[0]=0$; moreover combining (1.5) and the Sobolev's embeddings

$$
\begin{aligned}
J_{\varepsilon}[v] & \geq \frac{\varepsilon^{2}}{2} \int_{\Omega}|\nabla v|^{2} \mathrm{~d} x+\frac{1}{2} \int_{\Omega}|v|^{2} \mathrm{~d} x-C \int_{\Omega}|v|^{4} \mathrm{~d} x-C \int_{\Omega}|v|^{p+1} \mathrm{~d} x \\
& \geq\left(\frac{\varepsilon^{2}}{2}-C\|v\|_{H^{1}}^{2}-C\|v\|_{H^{1}}^{p-1}\right)\|v\|_{H^{1}}^{2} \geq \rho_{\varepsilon}>0
\end{aligned}
$$

provided that $\|v\|_{H^{1}}$ is sufficiently small. Condition (f4) can be restated as a differential equation for the function $F$ of the form $\frac{\mathrm{d}}{\mathrm{d} t} \log F \geq \frac{\theta}{t}$ for $t>0$, which implies

$$
F(t) \geq C\left(|t|^{\theta}-1\right) \quad \forall t \geq 0 .
$$

Hence, fixed $v \in H_{0}^{1}(\Omega) \backslash\{0\}$ such that $v_{+}=\max \{v, 0\} \neq 0$, we get

$$
\begin{aligned}
J_{\varepsilon}[\lambda v] \leq & \frac{\lambda^{2}}{2} \int_{\Omega}\left(\varepsilon^{2}|\nabla v|^{2}+|v|^{2}\right) \mathrm{d} x+\gamma \frac{\lambda^{4}}{4} \int_{\Omega} v^{2}(-\Delta)^{-1}\left[v^{2}\right] \mathrm{d} x \\
& -C \frac{\lambda^{\theta}}{4} \int_{\Omega}\left|v_{+}\right|^{\theta} \mathrm{d} x+C \rightarrow-\infty
\end{aligned}
$$

as $\lambda \rightarrow+\infty$. The well-known Mountain-Pass Lemma applies and gives that the following is actually a critical value for $J_{\varepsilon}$ :

$$
\tilde{J}_{\varepsilon}^{*}=\inf _{q \in \mathcal{Q}} \max _{t \in[0,1]} J_{\varepsilon}[q(t)]
$$

where $\mathcal{Q}=\left\{q \in C\left([0,1], H_{0}^{1}(\Omega)\right) \mid q(0)=0, J_{\varepsilon}[q(1)]<0\right\}$. Denoted by $v_{\varepsilon}$ the associated critical point, hence $v_{\varepsilon}$ solves the equation

$$
\varepsilon^{2} \Delta v_{\varepsilon}-v_{\varepsilon}+f\left(v_{\varepsilon}\right)-\gamma v_{\varepsilon}(-\Delta)^{-1}\left[v_{\varepsilon}^{2}\right]=0 \text { in } \Omega .
$$

It remains to show that $v_{\varepsilon}>0$. Indeed, multiplying (5.5) by $v_{\varepsilon}^{-}=\max \left\{0,-v_{\varepsilon}\right\}$, and using (f1) we see that

$$
\int_{\Omega}\left|\nabla v_{\varepsilon}^{-}\right|^{2} \mathrm{~d} x+\int_{\Omega}\left|v_{\varepsilon}^{-}\right|^{2} \mathrm{~d} x+\gamma \int_{\Omega}\left(v_{\varepsilon}^{-}\right)^{2}(-\Delta)^{-1}\left[v_{\varepsilon}^{2}\right] \mathrm{d} x=0
$$

which implies $v_{\varepsilon}^{-}=0$. By the strong maximum principle $v_{\varepsilon}>0$ in $\Omega$. Then $v_{\varepsilon}$ is a solution to (1.6).

We are going to prove that, as a consequence of condition (f2), we can characterize the values $\tilde{J}_{\varepsilon}^{*}$ in a simpler way, i.e. $\tilde{J}_{\varepsilon}^{*}=J_{\varepsilon}^{*}$. First notice that, since $J_{\varepsilon}[t v] \rightarrow+\infty$ for $v \in H_{0}^{1}(\Omega) \backslash\{0\}$ with $v_{+}=0$, then $J_{\varepsilon}^{*}$ can also be defined as

$$
J_{\varepsilon}^{*}=\inf _{v \in H_{0}^{1}(\Omega), v_{+} \neq 0} \sup _{t \geq 0} J_{\varepsilon}[t v] .
$$


For $v \in H_{0}^{1}(\Omega), v_{+} \neq 0$, we have $J_{\varepsilon}[\lambda v]<0$ for large $\lambda$, by which we immediately obtain $\tilde{J}_{\varepsilon}^{*} \leq J_{\varepsilon}^{*}$. In order to prove the opposite inequality, we first note that fixed $v \in H_{0}^{1}(\Omega)$ with $v_{+} \neq 0$, the function $\lambda>0 \mapsto J_{\varepsilon}[\lambda v]$ has a unique nontrivial critical point which is a maximum point. Indeed

$$
\begin{aligned}
& \frac{d}{d \lambda} J_{\varepsilon}[\lambda v]=\left\langle J_{\varepsilon}^{\prime}[\lambda v], v\right\rangle \\
& =\lambda^{3}\left(\frac{1}{\lambda^{2}} \int_{\Omega}\left(\varepsilon^{2}|\nabla v|^{2}+|v|^{2}\right) \mathrm{d} x+\gamma \int_{\Omega} v^{2}(-\Delta)^{-1}\left[v^{2}\right] \mathrm{d} x-\int_{\Omega} \frac{f(\lambda v)}{\lambda^{3} v^{3}} v^{4} \mathrm{~d} x\right) ;
\end{aligned}
$$

hypothesis (f2) implies that the bracket on the right hand side is a decreasing function of $\lambda$. Noting that, by (5.2), $J_{\varepsilon}[\lambda v]>0$ for $\lambda>0$ small and $J_{\varepsilon}[\lambda v] \rightarrow-\infty$ as $\lambda \rightarrow+\infty$, we obtain the assertion. Define $\lambda_{v}>0$ as the unique nontrivial critical point of $J_{\varepsilon}[\lambda v]$. Since $J_{\varepsilon}^{\prime}\left[v_{\varepsilon}\right]=0$, it is clear that $\frac{d}{d \lambda} J_{\varepsilon}\left[\lambda v_{\varepsilon}\right]_{\lambda=1}=0$, which implies $\lambda_{v_{\varepsilon}}=1$ and, consequently

$$
J_{\varepsilon}^{*} \leq J_{\varepsilon}\left[v_{\varepsilon}\right]=\tilde{J}_{\varepsilon}^{*} .
$$

Observe that

$$
J_{\varepsilon}^{*}=\inf _{v \in M_{\varepsilon}} J_{\varepsilon}[v]
$$

where

$$
\begin{aligned}
M_{\varepsilon}:=\left\{\lambda_{v} v \mid v \in H_{0}^{1}(\Omega), v_{+} \neq 0\right\} & \\
=\left\{v \in H_{0}^{1}(\Omega) \mid\left\langle J_{\varepsilon}^{\prime}[v], v\right\rangle:=\int_{\Omega}\left(\varepsilon^{2}|\nabla v|^{2}+|v|^{2}\right) \mathrm{d} x\right. & +\gamma \int_{\Omega} v^{2}(-\Delta)^{-1}\left[v^{2}\right] \mathrm{d} x \\
& \left.-\int_{\Omega} f(v) v \mathrm{~d} x=0\right\} .
\end{aligned}
$$

Since any nontrivial critical point of $J_{\varepsilon}$ lies on $M_{\varepsilon}$, then $J_{\varepsilon}^{*}$ is the smallest critical value of $J_{\varepsilon}$ and, consequently, $v_{\varepsilon}$ is a least-energy solution for (1.6).

To prove (5.1) choose $Q_{\varepsilon} \in \Omega$ such that $d_{\varepsilon}=d_{Q_{\varepsilon}}$ and $Q_{\varepsilon} \rightarrow P_{0}$ where $\mathcal{H}\left(P_{0}\right)=\mathcal{H}_{0}$. Then set $\lambda_{\varepsilon}=\lambda_{\tilde{w}_{\varepsilon, Q_{\varepsilon}}}>0$, i.e.

$$
\begin{aligned}
\lambda_{\varepsilon} \int_{\Omega}\left(\varepsilon^{2}\left|\nabla \tilde{w}_{\varepsilon, Q_{\varepsilon}}\right|^{2}+\left|\tilde{w}_{\varepsilon, Q_{\varepsilon}}\right|^{2}\right) \mathrm{d} x & +\lambda_{\varepsilon}^{3} \gamma \int_{\Omega} \tilde{w}_{\varepsilon, Q_{\varepsilon}}^{2}(-\Delta)^{-1}\left[\tilde{w}_{\varepsilon, Q_{\varepsilon}}^{2}\right] \mathrm{d} x \\
& -\int_{\Omega} f\left(\lambda_{\varepsilon} \tilde{w}_{\varepsilon, Q_{\varepsilon}}\right) \tilde{w}_{\varepsilon, Q_{\varepsilon}} \mathrm{d} x=0,
\end{aligned}
$$

which can be written, using Proposition 4.4, as

$$
\lambda_{\varepsilon} \int_{\Omega}\left(\varepsilon^{2}\left|\nabla \tilde{w}_{\varepsilon, Q_{\varepsilon}}\right|^{2}+\left|\tilde{w}_{\varepsilon, Q_{\varepsilon}}\right|^{2}\right) \mathrm{d} x+\mathrm{O}\left(\lambda_{\varepsilon}^{3} \varepsilon^{5}\right)-\int_{\Omega} f\left(\lambda_{\varepsilon} \tilde{w}_{\varepsilon, Q_{\varepsilon}}\right) \tilde{w}_{\varepsilon, Q_{\varepsilon}} \mathrm{d} x=0 .
$$


According to assumption (f2) we have $f(t) \geq c t^{3}$ for all $t \geq 0$; hence using (1.5) and Corollary 3.3 we get

$$
\frac{\varepsilon^{3} \int_{\mathbb{R}^{3}}\left(|\nabla w|^{2}+|w|^{2}\right) \mathrm{d} x+\mathrm{O}\left(\lambda_{\varepsilon}^{2} \varepsilon^{5}\right)}{C \varepsilon^{3}\left(\lambda_{\varepsilon}^{2} \int_{\mathbb{R}^{3}}|w|^{4} \mathrm{~d} x+\lambda_{\varepsilon}^{p-1} \int_{\mathbb{R}^{3}}|w|^{p+1} \mathrm{~d} x\right)} \leq \lambda_{\varepsilon} \leq \frac{\varepsilon^{3} \int_{\mathbb{R}^{3}}\left(|\nabla w|^{2}+|w|^{2}\right) \mathrm{d} x+\mathrm{O}\left(\lambda_{\varepsilon}^{2} \varepsilon^{5}\right)}{c \varepsilon^{3} \lambda_{\varepsilon}^{2} \int_{\mathbb{R}^{3}}|w|^{4} \mathrm{~d} x},
$$

by which $\left\{\lambda_{\varepsilon}\right\}$ turns out to be bounded from above and below away from 0 . We state that $\lambda_{\varepsilon} \rightarrow 1$ as $\varepsilon \rightarrow 0^{+}$. Indeed, assume by absurd that $\lambda_{\varepsilon_{n}} \rightarrow \bar{\lambda}>0$ and $\bar{\lambda} \neq 1$ for some sequence $\varepsilon_{n} \rightarrow 0^{+}$. Using Corollary 3.3 in (5.6) we get

$$
\bar{\lambda} \int_{\mathbb{R}^{3}}\left(|\nabla w|^{2}+|w|^{2}\right) \mathrm{d} x-\int_{\mathbb{R}^{3}} f(\bar{\lambda} w) w \mathrm{~d} x=0,
$$

i.e. $\bar{\lambda}$ is a critical point of $\lambda>0 \mapsto I[\lambda w]$. On the other hand $\lambda=1$ is obviously a critical point too, then the uniqueness of the nontrivial critical point (which can be proved as for $J_{\varepsilon}[\lambda w]$ ) gives the contradiction. Hence $\lambda_{\varepsilon} \rightarrow 1$ as $\varepsilon \rightarrow 0^{+}$. Using equation (3.1), (5.6) leads to

$$
\int_{\Omega} \lambda_{\varepsilon} f\left(w_{\varepsilon, Q_{\varepsilon}}\right) \tilde{w}_{\varepsilon, Q_{\varepsilon}} \mathrm{d} x-\int_{\Omega} f\left(\lambda_{\varepsilon} \tilde{w}_{\varepsilon, Q_{\varepsilon}}\right) \tilde{w}_{\varepsilon, Q_{\varepsilon}} \mathrm{d} x=\mathrm{O}\left(\varepsilon^{5}\right) .
$$

Observe that by assumption (f1) and (3.2) we get

$$
\begin{aligned}
\lambda_{\varepsilon} f\left(w_{\varepsilon, Q_{\varepsilon}}\right)-f\left(\lambda_{\varepsilon} \tilde{w}_{\varepsilon, Q_{\varepsilon}}\right)= & \left(\lambda_{\varepsilon}-1\right)\left(f\left(\tilde{w}_{\varepsilon, Q_{\varepsilon}}\right)-f^{\prime}\left(\tilde{w}_{\varepsilon, Q_{\varepsilon}}\right) \tilde{w}_{\varepsilon, Q_{\varepsilon}}+\mathrm{o}(1)\right) \\
& +\mathrm{O}\left(w_{\varepsilon, Q_{\varepsilon}}\left(\tilde{w}_{\varepsilon, Q_{\varepsilon}}-w_{\varepsilon, Q_{\varepsilon}}\right)\right)
\end{aligned}
$$

uniformly in $\Omega$. Hence Proposition 3.2 leads to

$$
\begin{aligned}
\mathrm{O}\left(\varepsilon^{5}\right)= & \left(\lambda_{\varepsilon}-1\right)\left(\int_{\Omega}\left(f\left(\tilde{w}_{\varepsilon, Q_{\varepsilon}}\right)-f^{\prime}\left(\tilde{w}_{\varepsilon, Q_{\varepsilon}}\right) \tilde{w}_{\varepsilon, Q_{\varepsilon}}\right) \tilde{w}_{\varepsilon, Q_{\varepsilon}} \mathrm{d} x+\mathrm{o}(1) \int_{\Omega} \tilde{w}_{\varepsilon, Q_{\varepsilon}} \mathrm{d} x\right) \\
& +\mathrm{O}\left(\int_{\Omega} w_{\varepsilon, Q_{\varepsilon}} w_{\varepsilon, Q_{\varepsilon}^{*}} \mathrm{~d} x\right)+\mathrm{O}\left(\int_{\Omega} w_{\varepsilon, Q_{\varepsilon}}\left(\varepsilon h_{\varepsilon, Q_{\varepsilon}}+k_{\varepsilon, Q_{\varepsilon}}\right) \mathrm{d} x\right)
\end{aligned}
$$

and then, combining (3.8), Corollary 3.3 and Lemma 4.1 we deduce

$$
\begin{aligned}
\mathrm{O}\left(\varepsilon^{2}\right)= & \left(\lambda_{\varepsilon}-1\right)\left(\int_{\mathbb{R}^{3}}\left(f(w)-f^{\prime}(w) w\right) w \mathrm{~d} x+\mathrm{o}(1)\right) \\
& +\mathrm{O}\left(e^{-2 \frac{d_{\varepsilon}}{\varepsilon}}\right)+\varepsilon^{-3} \mathrm{O}\left(\left\|w_{\varepsilon}, Q_{\varepsilon}\right\|_{L^{2}}\left\|\varepsilon h_{\varepsilon, Q_{\varepsilon}}+k_{\varepsilon, Q_{\varepsilon}}\right\|_{2}\right) \\
= & \left(\lambda_{\varepsilon}-1\right)\left(\int_{\mathbb{R}^{3}}\left(f(w)-f^{\prime}(w) w\right) w \mathrm{~d} x+\mathrm{o}(1)\right)+\mathrm{O}\left(\varepsilon^{5}+\varepsilon e^{-\frac{2 d_{\varepsilon}}{3 \varepsilon}}+e^{-2 \frac{d_{\varepsilon}}{\varepsilon}}\right) .
\end{aligned}
$$

In view of (f2) $\frac{f(t)}{t}$ is strictly increasing for $t>0$, hence $f(w)-f^{\prime}(w) w<0$. Thus we arrive at

$$
1-\lambda_{\varepsilon}=\mathrm{O}\left(\varepsilon^{2}+\varepsilon e^{-\frac{2 d_{\varepsilon}}{3 \varepsilon}}+e^{-2 \frac{d_{\varepsilon}}{\varepsilon}}\right) .
$$


Setting $\Lambda_{\varepsilon}(\lambda)=J_{\varepsilon}\left[\lambda \tilde{w}_{\varepsilon, Q_{\varepsilon}}\right]$, it is immediate that $\Lambda_{\varepsilon} \in \mathrm{C}^{2}$ and $\Lambda_{\varepsilon}^{\prime}\left(\lambda_{\varepsilon}\right)=0$; then, for some $t_{\varepsilon} \rightarrow 1$

$$
\begin{aligned}
J_{\varepsilon}^{*} \leq J_{\varepsilon}\left[\lambda_{\varepsilon} \tilde{w}_{\varepsilon, Q_{\varepsilon}}\right] & =\Lambda_{\varepsilon}\left(\lambda_{\varepsilon}\right)=\Lambda_{\varepsilon}(1)-\left(\lambda_{\varepsilon}-1\right)^{2} \Lambda_{\varepsilon}^{\prime \prime}\left(t_{\varepsilon}\right) \\
& =J_{\varepsilon}\left[\tilde{w}_{\varepsilon}, Q_{\varepsilon}\right]+\mathrm{O}\left(\varepsilon^{4}+\varepsilon^{2} e^{-\frac{4 d_{\varepsilon}}{3 \varepsilon}}+e^{-4 \frac{d_{\varepsilon}}{\varepsilon}}\right) \Lambda_{\varepsilon}^{\prime \prime}\left(t_{\varepsilon}\right)
\end{aligned}
$$

and the thesis follows from Propositions 4.3-4.4 once we have observed that

$$
\varepsilon^{-3} \Lambda_{\varepsilon}^{\prime \prime}\left(t_{\varepsilon}\right) \rightarrow \int_{\mathbb{R}^{3}}\left(|\nabla w|^{2}+|w|^{2}\right) \mathrm{d} x-\int_{\mathbb{R}^{3}} f^{\prime}(w) w^{2} \mathrm{~d} x
$$

Corollary 5.2. As a corollary of Theorem 5.3, choosing $d_{\varepsilon}=\varepsilon \log \frac{1}{\varepsilon}+\varepsilon \log \log \frac{1}{\varepsilon}$ and using (4.3), it is easy to verify that the main term in the envelope on the right hand side of (5.1) is represented by the first four; hence we obtain

$$
\begin{aligned}
& J_{\varepsilon}^{*} \leq I[w] \varepsilon^{3}+I_{2} \varepsilon^{5}-I_{3} \frac{\varepsilon^{5}}{|\log \varepsilon|+\log |\log \varepsilon|}+\varepsilon^{5} \frac{B(1+o(1))}{4|\log \varepsilon|^{2}(|\log \varepsilon|+\log |\log \varepsilon|)} \\
& \quad=I[w] \varepsilon^{3}+I_{2} \varepsilon^{5}-I_{3} \frac{\varepsilon^{5}}{|\log \varepsilon|}+o\left(\frac{\varepsilon^{5}}{|\log \varepsilon|^{5 / 3}}\right) \text { as } \varepsilon \rightarrow 0^{+}, \\
& \text {since } \frac{1}{|\log \varepsilon|+\log |\log \varepsilon|}-\frac{1}{|\log \varepsilon|}=\mathrm{O}\left(\frac{\log |\log \varepsilon|}{|\log \varepsilon|^{2}}\right)=\mathrm{o}\left(\frac{1}{|\log \varepsilon|^{5 / 3}}\right) .
\end{aligned}
$$

The goal of the next two propositions is to obtain more precise information on the shape of the least-energy solution $v_{\varepsilon}$; in particular we will prove that $v_{\varepsilon}$ develops a spike near the boundary whose profile resembles the approximation $\tilde{w}_{\varepsilon, P_{\varepsilon}}$ (for suitable $P_{\varepsilon} \in \Omega$ ) constructed in Section 3 up to the order $\mathrm{O}\left(\tau_{\varepsilon}\right)$ (for suitable $\tau_{\varepsilon} \rightarrow$ $\left.0^{+}\right)$.

Proposition 5.3. For $\varepsilon>0$ sufficiently small $v_{\varepsilon}$ is a single spike solution; more precisely there exists $P_{\varepsilon} \in \Omega$ such that $P_{\varepsilon}$ is a the maximum point of $v_{\varepsilon}$ on $\Omega$ and

$$
\frac{d_{P_{\varepsilon}}}{\varepsilon} \rightarrow+\infty \text { as } \varepsilon \rightarrow 0^{+}
$$

furthermore $v_{\varepsilon}$ satisfies

$$
v_{\varepsilon}=\tilde{w}_{\varepsilon, P_{\varepsilon}}+\psi_{\varepsilon}
$$

where

$$
\left\|\psi_{\varepsilon}\right\|_{\infty}+\varepsilon^{-3} \int_{\Omega}\left(\varepsilon^{2}\left|\nabla \psi_{\varepsilon}\right|^{2}+\left|\psi_{\varepsilon}\right|^{2}\right) \mathrm{d} x \rightarrow 0 \text { as } \varepsilon \rightarrow 0^{+}
$$


Proof. First we observe that, setting $u_{\varepsilon}(x)=v_{\varepsilon}(\varepsilon x)$ for $x \in \frac{\Omega}{\varepsilon}$ and $u_{\varepsilon}=0$ in $\mathbb{R}^{3} \backslash \frac{\Omega}{\varepsilon}$, by assumption (f4) we have

$$
\begin{aligned}
\left(\frac{1}{2}-\frac{1}{\theta}\right) \int_{\mathbb{R}^{3}}\left(\left|\nabla u_{\varepsilon}\right|^{2}+\left|u_{\varepsilon}\right|^{2}\right) \mathrm{d} x & =\varepsilon^{-3}\left(\frac{1}{2}-\frac{1}{\theta}\right) \int_{\Omega}\left(\varepsilon^{2}\left|\nabla v_{\varepsilon}\right|^{2}+\left|v_{\varepsilon}\right|^{2}\right) \mathrm{d} x \\
& \leq \varepsilon^{-3} J_{\varepsilon}\left[v_{\varepsilon}\right]-\frac{\varepsilon^{-3}}{\theta}\left\langle J_{\varepsilon}^{\prime}\left[v_{\varepsilon}\right], v_{\varepsilon}\right\rangle=\varepsilon^{-3} J_{\varepsilon}\left[v_{\varepsilon}\right] \\
& =\varepsilon^{-3} J_{\varepsilon}^{*}=I[w]+\mathrm{O}\left(\varepsilon^{2}\right) \text { as } \varepsilon \rightarrow 0^{+},
\end{aligned}
$$

where in the last inequality we have used Corollary 5.2. Then $\left\{u_{\varepsilon}\right\}$ is bounded in $H^{1}\left(\mathbb{R}^{3}\right)$ for small $\varepsilon$. For the sake of simplicity we divide the remaining part of the proof into four claims.

Claim 1. For every $R>0$ and $1 \leq q<6$ :

$$
\lim _{\varepsilon \rightarrow 0^{+}} \sup _{d_{P} \leq \varepsilon R} \int_{B\left(\frac{P}{\varepsilon}, R\right)} u_{\varepsilon}^{q} \mathrm{~d} x=0 .
$$

For otherwise, there exist $q \in[1,6), R>0$, a sequence $u_{n}:=u_{\varepsilon_{n}}$ and $P_{n} \in \mathbb{R}^{3}$ with $d_{P_{n}} \leq \varepsilon_{n} R$ such that

$$
\lim _{n \rightarrow+\infty} \int_{B\left(\frac{P_{n}}{\varepsilon_{n}}, R\right)} u_{n}^{q} \mathrm{~d} x>0
$$

Then set $\tilde{u}_{n}(y)=u_{n}\left(x+\frac{P_{n}}{\varepsilon_{n}}\right)$ where $y=T_{P_{n}} x$ and, without loss of generality, we may assume $\operatorname{dist}\left(\frac{P_{n}}{\varepsilon_{n}}, \frac{\partial \Omega}{\varepsilon_{n}}\right)=\frac{d P_{n}}{\varepsilon_{n}} \rightarrow \delta \in[0, R]$ and $\tilde{u}_{n} \rightarrow \tilde{u}$ weakly in $H^{1}\left(\mathbb{R}^{3}\right)$. Since by construction $\chi_{\frac{1}{\varepsilon_{n}}} T_{P_{n}} \Omega \rightarrow \chi_{\mathbb{R}_{+}^{3}}$ (denoting $\chi$ the characteristic function), then, setting $\mathbb{R}_{\delta,+}^{3}=\left\{y \in \mathbb{R}^{3} \mid y_{n}>-\delta\right\}$, we deduce $\tilde{u} \in H_{0}^{1}\left(\mathbb{R}_{\delta,+}^{3}\right)$ and (since by b) of Proposition $\left.2.1(-\Delta)^{-1}\left[v_{\varepsilon}^{2}\right] \leq C\left\|v_{\varepsilon}\right\|_{L^{4}}^{2}=\mathrm{O}\left(\varepsilon^{3 / 2}\right)\right) \tilde{u}$ solves

$$
\Delta \tilde{u}-\tilde{u}+f(\tilde{u})=0 \text { in } \mathbb{R}_{\delta,+}^{3}, \quad \tilde{u} \geq 0 \text { in } \mathbb{R}_{\delta,+}^{3}, \quad \tilde{u}=0 \text { on } \partial \mathbb{R}_{\delta,+}^{3} .
$$

Theorem 1.1 in [20] implies $\tilde{u}=0$, which is a contradiction since

$$
\int_{B(0, R)} \tilde{u}^{q} \mathrm{~d} x=\lim _{n \rightarrow+\infty} \int_{B(0, R)} \tilde{u}_{n}^{q} \mathrm{~d} x=\lim _{n \rightarrow+\infty} \int_{B\left(\frac{P_{n}}{\varepsilon_{n}}, R\right)} u_{n}^{2} \mathrm{~d} x>0 .
$$

Hence Claim 1 holds.

Claim 2. There exists $\eta>0$ such that if $\varepsilon \in(0,1)$ and $Q_{\varepsilon}$ is a local maximum point for $v_{\varepsilon}$, then

$$
v_{\varepsilon}\left(Q_{\varepsilon}\right) \geq \eta
$$


Indeed

$$
\begin{aligned}
v_{\varepsilon}\left(Q_{\varepsilon}\right)-f\left(v_{\varepsilon}\left(Q_{\varepsilon}\right)\right) & \leq v_{\varepsilon}\left(Q_{\varepsilon}\right)+v_{\varepsilon}\left(Q_{\varepsilon}\right)(-\Delta)^{-1}\left[v_{\varepsilon}^{2}\right]\left(Q_{\varepsilon}\right)-f\left(v_{\varepsilon}\left(Q_{\varepsilon}\right)\right) \\
& =\varepsilon^{2} \Delta v_{\varepsilon}\left(Q_{\varepsilon}\right) \leq 0
\end{aligned}
$$

Using (1.5) we obtain the thesis.

Claim 3. For every $R>0$

$$
\limsup _{\varepsilon \rightarrow 0^{+}} \sup _{d_{P} \leq \varepsilon R} v_{\varepsilon}(P)=0 .
$$

Fix $R>0$ sufficiently large such that

$$
\frac{1}{2 \pi R} \int_{\mathbb{R}^{3}} f\left(u_{n}\right) \mathrm{d} x \leq \frac{\eta}{2}
$$

and let $P_{\varepsilon} \in \Omega$ be the maximum point for $v_{\varepsilon}$ in $\left\{x \in \Omega \mid d_{P} \leq \varepsilon R\right\}$. Assume by absurd that there is a sequence $\varepsilon_{n} \rightarrow 0$ such that $v_{n}\left(P_{n}\right):=v_{\varepsilon_{n}}\left(P_{\varepsilon_{n}}\right) \nrightarrow 0$. First observe that $\frac{d_{P_{n}}}{\varepsilon_{n}} \rightarrow 0$. Otherwise, up to a subsequence, $\frac{d_{P_{n}}}{\varepsilon_{n}} \rightarrow \delta>0$, and, proceeding as in Claim 1, setting $\tilde{u}_{n}(y)=u_{n}\left(x+\frac{P_{n}}{\varepsilon_{n}}\right)$ with $y=T_{P_{n}}(x)$, we deduce $\tilde{u}_{n} \rightarrow 0$ weakly in $H^{1}\left(\mathbb{R}^{3}\right)$. On the other hand by standard elliptic regularity we have $\tilde{u}_{n} \rightarrow 0$ uniformly on compact sets of $\mathbb{R}_{\delta,+}^{3}$, then $v_{n}\left(P_{n}\right)=\tilde{u}_{n}(0) \rightarrow 0$ which is a contradiction. Hence $\frac{d P_{n}}{\varepsilon_{n}} \rightarrow 0$, which implies that, at least for large $n, P_{n}$ is a local maximum point for $v_{n}$ in $B\left(P_{n}, \varepsilon_{n} \frac{R}{2}\right)$. Consequently, by Claim $2, v_{n}\left(P_{n}\right) \geq \eta$ and, by (1.5), $\frac{f\left(u_{n}\right)}{v_{n}\left(P_{n}\right)} \leq C\left(\left|u_{n}\right|^{2}+\left|u_{n}\right|^{p-1}\right)$ for $\left|z-\frac{P_{n}}{\varepsilon_{n}}\right| \leq \frac{R}{2}$.

By using the comparison principle it is immediate that $0 \leq u_{\varepsilon} \leq w_{\varepsilon}$ where $w_{\varepsilon}$ solves

$$
\Delta w_{\varepsilon}+f\left(u_{\varepsilon}\right)=0 \text { in } \frac{\Omega}{\varepsilon}, \quad w_{\varepsilon}=0 \text { on } \frac{\partial \Omega}{\varepsilon} .
$$

Fix $\frac{3}{2}<a<\frac{6}{p-1}$ and let $a^{\prime}<3$ be such that $\frac{1}{a}+\frac{1}{a^{\prime}}=1$; by (2.1), using Hölder's inequality, we get

$$
\begin{aligned}
\eta & \leq v_{n}\left(P_{n}\right) \leq w_{\varepsilon_{n}}\left(\frac{P_{n}}{\varepsilon_{n}}\right) \leq \frac{1}{4 \pi} \int_{\frac{\Omega}{\varepsilon_{n}}} \frac{f\left(u_{n}\right)}{\left|\frac{P_{n}}{\varepsilon_{n}}-z\right|} \mathrm{d} z \\
& \leq \frac{1}{4 \pi} \int_{\left|z-\frac{P_{n}}{\varepsilon_{n}}\right| \leq \frac{R}{2}} \frac{f\left(u_{n}\right)}{\left|\frac{P_{n}}{\varepsilon_{n}}-z\right|} \mathrm{d} z+\frac{1}{2 \pi R} \int_{\mathbb{R}^{3}} f\left(u_{n}\right) \mathrm{d} z \\
& \leq C v_{n}\left(P_{n}\right) \int_{\left|z-\frac{P_{n}}{\varepsilon_{n}}\right| \leq \frac{R}{2}} \frac{\left|u_{n}\right|^{2}+\left|u_{n}\right|^{p-1}}{\left|\frac{P_{n}}{\varepsilon_{n}}-z\right|} \mathrm{d} z+\frac{\eta}{2} \\
& \leq C v_{n}\left(P_{n}\right)\left(\int_{|z| \leq \frac{R}{2}} \frac{1}{|z|^{a^{\prime}}} \mathrm{d} z\right)^{1 / a^{\prime}}\left(\int_{\left|z-\frac{P_{n}}{\varepsilon_{n}}\right| \leq \frac{R}{2}}\left(\left|u_{n}\right|^{2 a}+\left|u_{n}\right|^{a(p-1)}\right) \mathrm{d} z\right)^{1 / a}+\frac{\eta}{2} ;
\end{aligned}
$$


then Claim 1 implies

$$
\int_{\left|z-\frac{P_{n}}{\varepsilon_{n}}\right| \leq \frac{R}{2}}\left|u_{n}\right|^{2 a} \mathrm{~d} z, \quad \int_{\left|z-\frac{P_{n}}{\varepsilon_{n}}\right| \leq \frac{R}{2}}\left|u_{n}\right|^{a(p-1)} \mathrm{d} z \rightarrow 0 .
$$

Hence we deduce

$$
\eta \leq v_{n}\left(P_{n}\right) \leq o\left(v_{n}\left(P_{n}\right)\right)+\frac{\eta}{2} .
$$

by which $\eta \leq v_{n}\left(P_{n}\right) \leq \mathrm{o}(1)+\frac{\eta}{2}$ and the contradiction follows.

Claim 4. End of the proof.

Let $P_{\varepsilon}$ be the maximum point for $v_{\varepsilon}$ in $\Omega$. According to Claims 2 and 3 $v_{\varepsilon}\left(P_{\varepsilon}\right) \geq \eta$ and $\frac{d_{P_{\varepsilon}}}{\varepsilon} \rightarrow+\infty$ as $\varepsilon \rightarrow 0^{+}$. Let $\varepsilon_{n} \rightarrow 0^{+}$be an arbitrary sequence and set $P_{n}=P_{\varepsilon_{n}}$ and $\bar{u}_{n}(x)=u_{\varepsilon_{n}}\left(x+\frac{P_{n}}{\varepsilon_{n}}\right)$. Since $\operatorname{dist}\left(\frac{P_{n}}{\varepsilon_{n}}, \frac{\partial \Omega}{\varepsilon_{n}}\right)=\frac{d_{P_{n}}}{\varepsilon_{n}} \rightarrow+\infty$, we may assume $\bar{u}_{n} \rightarrow \bar{u}$ weakly in $H^{1}\left(\mathbb{R}^{3}\right)$ where $\bar{u}$ solves

$$
\Delta \bar{u}-\bar{u}+f(\bar{u})=0 \text { in } \mathbb{R}^{3} .
$$

The elliptic regularity theory implies $\lim _{|x| \rightarrow+\infty} \bar{u}=0$ (see [21], Theorem 5, and [42]) and $\bar{u}_{n} \rightarrow \bar{u}$ in $C_{\mathrm{loc}}^{1}\left(\mathbb{R}^{3}\right)$; in particular $\bar{u}(0)=\max _{x \in \mathbb{R}^{3}} \bar{u}(x) \geq \eta$ and $\bar{u} \geq 0$, consequently, from the strong maximum principle $\bar{u}>0$ in $\mathbb{R}^{3}$. Assumption (f5) implies $\bar{u}=w$. Then by Fatou's Lemma we get

$$
\begin{aligned}
\varepsilon_{n}^{-3} \int_{\Omega}\left(\frac{1}{\theta} f\left(v_{\varepsilon_{n}}\right) v_{\varepsilon_{n}}-F\left(v_{\varepsilon_{n}}\right)\right) \mathrm{d} x & =\int_{\mathbb{R}^{3}}\left(\frac{1}{\theta} f\left(\bar{u}_{n}\right) \bar{u}_{n}-F\left(\bar{u}_{n}\right)\right) \\
& \geq \int_{\mathbb{R}^{3}}\left(\frac{1}{\theta} f(w) w-F(w)\right) \mathrm{d} x+\mathrm{o}(1),
\end{aligned}
$$

by which, since $J_{\varepsilon}^{\prime}\left[v_{\varepsilon}\right]=I^{\prime}[w]=0$, using Corollary 5.2,

$$
\begin{aligned}
& \left(\frac{1}{2}-\frac{1}{\theta}\right) \int_{\mathbb{R}^{3}}\left(\left|\nabla \bar{u}_{n}\right|^{2}+\left|\bar{u}_{n}\right|^{2}\right) \mathrm{d} x=\varepsilon_{n}^{-3}\left(\frac{1}{2}-\frac{1}{\theta}\right) \int_{\Omega}\left(\varepsilon^{2}\left|\nabla v_{\varepsilon_{n}}\right|^{2}+\left|v_{\varepsilon_{n}}\right|^{2}\right) \mathrm{d} x \\
& \leq \varepsilon_{n}^{-3} J_{\varepsilon_{n}}\left[v_{\varepsilon_{n}}\right]-\frac{\varepsilon_{n}^{-3}}{\theta}\left\langle J_{\varepsilon_{n}}^{\prime}\left[v_{\varepsilon_{n}}\right], v_{\varepsilon_{n}}\right\rangle-\varepsilon_{n}^{-3} \int_{\Omega}\left(\frac{1}{\theta} f\left(v_{\varepsilon_{n}}\right) v_{\varepsilon_{n}}-F\left(v_{\varepsilon_{n}}\right)\right) \mathrm{d} x \\
& \leq I[w]-\int_{\mathbb{R}^{3}}\left(\frac{1}{\theta} f(w) w-F(w)\right) \mathrm{d} x+\mathrm{o}(1) \\
& =I[w]-\frac{1}{\theta}\left\langle I^{\prime}[w], w\right\rangle-\int_{\mathbb{R}^{3}}\left(\frac{1}{\theta} f(w) w-F(w)\right) \mathrm{d} x+\mathrm{o}(1) \\
& =\left(\frac{1}{2}-\frac{1}{\theta}\right) \int_{\mathbb{R}^{3}}\left(|\nabla w|^{2}+|w|^{2}\right) \mathrm{d} x+\mathrm{o}(1) .
\end{aligned}
$$


The weakly lower semicontinuity of the norm implies the opposite inequality, hence $\int_{\mathbb{R}^{3}}\left(\left|\nabla \bar{u}_{n}\right|^{2}+\left|\bar{u}_{n}\right|^{2}\right) \mathrm{d} x \rightarrow \int_{\mathbb{R}^{3}}\left(|\nabla w|^{2}+|w|^{2}\right) \mathrm{d} x$, which implies $\bar{u}_{n} \rightarrow w$ in $H^{1}\left(\mathbb{R}^{3}\right)$. The arbitrariness of $\varepsilon_{n}$ leads to

$$
u_{\varepsilon}\left(\cdot+\frac{P_{\varepsilon}}{\varepsilon}\right) \rightarrow w \text { in } H^{1}\left(\mathbb{R}^{3}\right) \text { and } C_{\mathrm{loc}}^{1}\left(\mathbb{R}^{3}\right) .
$$

It remains to prove that

$$
\lim _{|x| \rightarrow+\infty} u_{\varepsilon}\left(\cdot+\frac{P_{\varepsilon}}{\varepsilon}\right)=0 \text { uniformly with respect to } \varepsilon .
$$

For otherwise there would be a sequence $\varepsilon_{n} \rightarrow 0, Q_{n} \in \Omega$ with $\frac{\left|Q_{n}-P_{n}\right|}{\varepsilon_{n}} \rightarrow+\infty$ and $u_{\varepsilon_{n}}\left(\frac{Q_{n}}{\varepsilon_{n}}\right) \geq c>0$. According to Claim $3 \frac{d_{Q_{n}}}{\varepsilon_{n}} \rightarrow+\infty$ as $n \rightarrow+\infty$, hence $u_{\varepsilon_{n}}\left(x+\frac{Q_{n}}{\varepsilon_{n}}\right) \rightarrow u$ in $H^{1}\left(\mathbb{R}^{3}\right)$ and in $C_{\text {loc }}^{1}\left(\mathbb{R}^{3}\right)$ (with $u$ solving (5.9)), by which $u(0)=\lim u_{\varepsilon_{n}}\left(\frac{Q_{n}}{\varepsilon_{n}}\right) \geq c$; in particular $u \neq 0$, which is in contradiction with (5.10). Hence we have proved that $u_{\varepsilon}\left(\cdot+\frac{P_{\varepsilon}}{\varepsilon}\right) \rightarrow w$ in $H^{1}\left(\mathbb{R}^{3}\right)$ and $L^{\infty}\left(\mathbb{R}^{3}\right)$, which implies, using Corollary 3.3, that $\psi_{\varepsilon}(\varepsilon x):=u_{\varepsilon}(x)-\tilde{w}_{\varepsilon, P_{\varepsilon}}(\varepsilon x) \rightarrow 0$ in $H^{1}\left(\mathbb{R}^{3}\right)$ and $L^{\infty}\left(\mathbb{R}^{3}\right)$.

In the last proposition of this section we go further in the analysis began in the previous one and provide an estimate on the error order up to the approximation $\tilde{w}_{\varepsilon}, P_{\varepsilon}$ works in the expansion of $v_{\varepsilon}$.

Proposition 5.4. For $\varepsilon>0$ sufficiently small $v_{\varepsilon}$ satisfies

$$
v_{\varepsilon}=\tilde{w}_{\varepsilon, P_{\varepsilon}}+\tau_{\varepsilon} \phi_{\varepsilon}
$$

where $\tau_{\varepsilon}=\max \left\{e^{-\frac{5 d_{\varepsilon}}{3 \varepsilon}}, \varepsilon e^{-\frac{3 d_{\varepsilon}}{5 \varepsilon}}, \varepsilon^{5 / 3}\right\}$ and, for $\varepsilon$ sufficiently small, $\phi_{\varepsilon}$ verifies

$$
\left\|\phi_{\varepsilon}\right\|_{\infty}+\varepsilon^{-3} \int_{\Omega}\left(\varepsilon^{2}\left|\nabla \phi_{\varepsilon}\right|^{2}+\left|\phi_{\varepsilon}\right|^{2}\right) \mathrm{d} x \leq C .
$$

Proof. Substituting $v_{\varepsilon}=\tilde{w}_{\varepsilon, P_{\varepsilon}}+\tau_{\varepsilon} \phi_{\varepsilon}$ into the equation $\mathcal{S}_{\varepsilon}\left[v_{\varepsilon}\right]=0$ we obtain

$$
\varepsilon^{2} \Delta \phi_{\varepsilon}-\phi_{\varepsilon}+f^{\prime}\left(\tilde{w}_{\varepsilon, P_{\varepsilon}}\right) \phi_{\varepsilon}=-\tau_{\varepsilon}^{-1} \mathcal{S}_{\varepsilon}\left[\tilde{w}_{\varepsilon, P_{\varepsilon}}\right]+N_{\varepsilon}\left[\phi_{\varepsilon}\right]+M_{\varepsilon}\left[\phi_{\varepsilon}\right],
$$

where

$$
\begin{aligned}
N_{\varepsilon}[\phi]= & -\tau_{\varepsilon}^{-1}\left(f\left(\tilde{w}_{\varepsilon, P_{\varepsilon}}+\tau_{\varepsilon} \phi\right)-f\left(\tilde{w}_{\varepsilon, P_{\varepsilon}}\right)-\tau_{\varepsilon} f^{\prime}\left(\tilde{w}_{\varepsilon, P_{\varepsilon}}\right) \phi\right), \\
M_{\varepsilon}[\phi]= & \gamma \tau_{\varepsilon}\left(\tilde{w}_{\varepsilon, P_{\varepsilon}}+\tau_{\varepsilon} \phi\right)(-\Delta)^{-1}\left[\phi^{2}\right]+2 \gamma\left(\tilde{w}_{\varepsilon, P_{\varepsilon}}+\tau_{\varepsilon} \phi\right)(-\Delta)^{-1}\left[\tilde{w}_{\varepsilon, P_{\varepsilon}} \phi\right] \\
& +\gamma(-\Delta)^{-1}\left[\tilde{w}_{\varepsilon, P_{\varepsilon}}^{2}\right] \phi .
\end{aligned}
$$


By Lemma 3.4 for $\varepsilon$ sufficiently small we have

$$
\left|\tau_{\varepsilon}^{-1} \mathcal{S}_{\varepsilon}\left[\tilde{w}_{\varepsilon, P_{\varepsilon}}\right]\right| \leq C \max \left\{e^{-\frac{d_{\varepsilon}}{12 \varepsilon}}, e^{-\frac{d_{\varepsilon}}{15 \varepsilon}}, \varepsilon^{1 / 3}\right\} w_{\varepsilon, P_{\varepsilon}}^{1 / 4} \quad \text { uniformly in } \Omega
$$

while, since by Proposition $5.3\left\|\tau_{\varepsilon} \phi_{\varepsilon}\right\|_{\infty}=\left\|\psi_{\varepsilon}\right\|_{\infty}=\mathrm{o}(1),\left\|\tau_{\varepsilon} \phi_{\varepsilon}\right\|_{L^{2}}^{2}=\left\|\psi_{\varepsilon}\right\|_{L^{2}}^{2}=$ $\mathrm{o}\left(\varepsilon^{3}\right)$, by the mean value theorem we get

$$
\left|N_{\varepsilon}\left[\phi_{\varepsilon}\right]\right| \leq C \tau_{\varepsilon} \phi_{\varepsilon}^{2}=\mathrm{o}(1)\left|\phi_{\varepsilon}\right| \quad \text { uniformly in } \Omega
$$

and, using c) of Proposition 2.1, we have $\tau_{\varepsilon}(-\Delta)^{-1}\left[\phi_{\varepsilon}^{2}\right], \tau_{\varepsilon}\left|(-\Delta)^{-1}\left[\tilde{w}_{\varepsilon, P_{\varepsilon}} \phi_{\varepsilon}\right]\right|=$ $\mathrm{o}\left(\varepsilon^{2} \tau_{\varepsilon}^{-1}\right)=\mathrm{o}(1)$ and $\left|(-\Delta)^{-1}\left[\tilde{w}_{\varepsilon, P_{\varepsilon}}^{2}\right]\right|=\mathrm{O}\left(\varepsilon^{2}\right)$ by which

$$
\left|M_{\varepsilon}\left[\phi_{\varepsilon}\right]\right|=\mathrm{o}(1) w_{\varepsilon}, P_{\varepsilon}+\mathrm{o}(1)\left|\phi_{\varepsilon}\right|,
$$

uniformly in $\Omega$. Suppose that there exists a sequence $\varepsilon_{n} \rightarrow 0^{+}$such that, setting $\phi_{n}:=\phi_{\varepsilon_{n}},\left\|\phi_{n}\right\|_{\infty} \rightarrow+\infty$ and let $x_{n} \in \Omega$ be such that $\left|\phi_{n}\left(x_{n}\right)\right|=\left\|\phi_{n}\right\|_{\infty}$. We may assume, without loss of generality, that $x_{n}$ is a maximum. Then we claim that

$$
\frac{\left|x_{n}-P_{\varepsilon_{n}}\right|}{\varepsilon_{n}} \leq C
$$

Otherwise, up to a subsequence, $\frac{\left|x_{n}-P_{\varepsilon_{n}}\right|}{\varepsilon_{n}} \rightarrow+\infty$. Then by (3.2) we have $\tilde{w}_{\varepsilon_{n}, P_{\varepsilon_{n}}}\left(x_{n}\right) \rightarrow 0$, and, consequently, $f^{\prime}\left(\tilde{w}_{\varepsilon_{n}, P_{\varepsilon_{n}}}\left(x_{n}\right)\right) \rightarrow 0$, while $\Delta \phi_{n}\left(x_{n}\right) \leq 0$. Combining (5.12)-(5.15) we arrive at

$$
0=\varepsilon_{n}^{2} \Delta \phi_{n}\left(x_{n}\right)-(1+\mathrm{o}(1))\left\|\phi_{n}\right\|_{\infty}+\mathrm{o}(1) \leq-(1+\mathrm{o}(1))\left\|\phi_{n}\right\|_{\infty}+\mathrm{o}(1)
$$

and hence $\left\|\phi_{n}\right\|_{\infty}=\mathrm{o}(1)$, in contrast to the hypothesis. Then (5.16) holds, and we may assume $\frac{x_{n}-P_{\varepsilon_{n}}}{\varepsilon_{n}} \rightarrow \bar{x}$. Now set $\hat{\phi}_{n}(x)=\frac{\phi_{n}}{\left\|\phi_{n}\right\|_{\infty}}\left(\varepsilon_{n} x+P_{\varepsilon_{n}}\right)$ for $x \in \frac{\Omega-P_{\varepsilon_{n}}}{\varepsilon_{n}}$. By dividing both members of (5.12) by $\left\|\phi_{n}\right\|_{\infty}$ and using (5.13)-(5.15), we deduce that $\hat{\phi}_{n}$ solves

$$
\begin{aligned}
\Delta \hat{\phi}_{n}-\hat{\phi}_{n}+f^{\prime}\left(\tilde{w}_{\varepsilon, P_{\varepsilon_{n}}}\left(\varepsilon_{n} x+P_{\varepsilon_{n}}\right)\right) \hat{\phi}_{n} & =\mathrm{o}(1)\left|\hat{\phi}_{n}\right|+\mathrm{o}(1) w^{1 / 4}, \\
\left|\hat{\phi}_{n}\right| & \leq 1 \text { in } \frac{\Omega-P_{\varepsilon_{n}}}{\varepsilon_{n}} .
\end{aligned}
$$

By multiplying both members of (5.17) by $\hat{\phi}_{n}$ we get

$$
\begin{aligned}
\int_{\mathbb{R}^{3}}\left(\left|\nabla \hat{\phi}_{n}\right|^{2}+\left|\hat{\phi}_{n}\right|^{2}\right) \mathrm{d} x & \leq \int_{\mathbb{R}^{3}}\left(\left|f^{\prime}\left(\tilde{w}_{\varepsilon_{n}, P_{\varepsilon_{n}}}\right)\left(\varepsilon_{n} x\right)\right|+w^{1 / 4}\right) \mathrm{d} x+\mathrm{o}(1) \int_{\mathbb{R}^{3}}\left|\hat{\phi}_{n}\right|^{2} \mathrm{~d} x \\
& \leq C+\mathrm{o}(1) \int_{\mathbb{R}^{3}}\left|\hat{\phi}_{n}\right|^{2} \mathrm{~d} x,
\end{aligned}
$$


i.e. $\hat{\phi}_{n}$ is bounded in $H^{1}\left(\mathbb{R}^{3}\right)$. Hence we may assume $\hat{\phi}_{n} \rightarrow \hat{\phi}$ weakly in $H^{1}\left(\mathbb{R}^{3}\right)$. Since by Proposition $5.3 \frac{d P_{\varepsilon}}{\varepsilon} \rightarrow+\infty$ we get $\frac{\chi \Omega-P_{\varepsilon_{n}}}{\varepsilon_{n}} \rightarrow \chi_{\mathbb{R}^{3}}=1$; then, by Corollary $3.3, \hat{\phi}$ solves

$$
\Delta \hat{\phi}-\hat{\phi}+f^{\prime}(w) \hat{\phi}=0, \quad|\hat{\phi}| \leq 1 \text { in } \mathbb{R}^{3} .
$$

Furthermore elliptic estimates lead to $\hat{\phi} \in H^{2}\left(\mathbb{R}^{3}\right)$ and $\hat{\phi}_{n} \rightarrow \hat{\phi}$ in $C_{\text {loc }}^{1}\left(\mathbb{R}^{3}\right)$, which implies $\hat{\phi}(\bar{x})=\lim _{n} \hat{\phi}_{n}\left(\frac{x_{n}-P_{\varepsilon_{n}}}{\varepsilon_{n}}\right)=\lim _{n} \frac{\phi_{n}\left(x_{n}\right)}{\left\|\phi_{n}\right\|_{\infty}}=1$. In particular $\hat{\phi} \neq 0$. Assumption (f5) leads to

$$
\hat{\phi}=\sum_{j=1}^{3} a_{j} \frac{\partial w}{\partial x_{j}}
$$

for some constants $a_{1}, a_{2}, a_{3}$. On the other hand by Part iii) of Proposition 3.2 and the choice of $\tau_{\varepsilon}$ we have $\varepsilon \nabla \tilde{w}_{\varepsilon, P_{\varepsilon}}\left(P_{\varepsilon}\right)=\mathrm{o}\left(\tau_{\varepsilon}\right)$, by which

$$
0=\nabla v_{\varepsilon_{n}}\left(P_{\varepsilon_{n}}\right)=\nabla\left(\tilde{w}_{\varepsilon_{n}, P_{\varepsilon_{n}}}+\tau_{\varepsilon_{n}} \phi_{n}\right)\left(P_{\varepsilon_{n}}\right)=\mathrm{o}\left(\varepsilon_{n}^{-1} \tau_{\varepsilon_{n}}\right)+\tau_{\varepsilon_{n}} \varepsilon_{n}^{-1}\left\|\phi_{n}\right\|_{\infty} \nabla \hat{\phi}_{n}(0) .
$$

This implies $\nabla \hat{\phi}(0)=0$, i.e. $\left(\right.$ since $\frac{\partial^{2} w}{\partial x_{k} \partial x_{j}}(0)=0$ if $j \neq k$ and $\frac{\partial^{2} w}{\partial x_{j}^{2}}(0)=w^{\prime \prime}(0)$ ) $a_{j} w^{\prime \prime}(0)=0$. The contradiction will follow if we prove that $w^{\prime \prime}(0) \neq 0$. Otherwise, from assumption (f5) $w(0)=f(w(0))$. By (f2) $\frac{f(t)}{t}$ is strictly increasing for $t>0$, while $w(0)=\max _{\mathbb{R}^{3}} w$; hence $w-f(w) \geq 0$ in $\mathbb{R}^{3}$. This is a contradiction since $\int_{\mathbb{R}^{3}}|\nabla w|^{2}+\int_{\mathbb{R}^{3}}(w-f(w)) w=0$. Thus $\left|\phi_{\varepsilon}\right| \leq C$ for small $\varepsilon$. Finally, multiplying the equation (5.12) by $\phi_{\varepsilon}$, integrating over $\Omega$ and using (5.13)-(5.15), we obtain

$$
\begin{aligned}
& \varepsilon^{2} \int_{\Omega}\left|\nabla \phi_{\varepsilon}\right|^{2} \mathrm{~d} x+\int_{\Omega}\left|\phi_{\varepsilon}\right|^{2} \mathrm{~d} x \\
& \quad \leq \int_{\Omega} f^{\prime}\left(\tilde{w}_{\varepsilon, P_{\varepsilon}}\right) \phi_{\varepsilon}^{2} \mathrm{~d} x+\mathrm{o}(1) \int_{\Omega} \phi_{\varepsilon} w_{\varepsilon, P_{\varepsilon}}^{1 / 4} \mathrm{~d} x+\mathrm{o}(1) \int_{\Omega}\left|\phi_{\varepsilon}\right|^{2} \mathrm{~d} x \\
& \quad \leq C \int_{\Omega} f^{\prime}\left(\tilde{w}_{\varepsilon, P_{\varepsilon}}\right) \mathrm{d} x+\mathrm{o}(1) \int_{\Omega} w_{\varepsilon, P_{\varepsilon}}^{1 / 4} \mathrm{~d} x+\mathrm{o}(1) \int_{\Omega}\left|\phi_{\varepsilon}\right|^{2} \mathrm{~d} x \\
& \quad \leq C \varepsilon^{-3}+\mathrm{o}(1) \int_{\Omega}\left|\phi_{\varepsilon}\right|^{2} \mathrm{~d} x .
\end{aligned}
$$

\section{Proof of Theorem 1.1}

Now we have all the ingredients to prove Theorem 1.1. We just have to combine the results of Proposition 5.3 and 5.4. In what follows we write $\hat{d}_{\varepsilon}$ in place of $d_{P_{\varepsilon}}$. 
Step 1. The following holds:

$$
\begin{aligned}
J_{\varepsilon}^{*} & =J_{\varepsilon}\left[v_{\varepsilon}\right]=J_{\varepsilon}\left[\tilde{w}_{\varepsilon, P_{\varepsilon}}\right]+\mathrm{O}\left(\varepsilon^{3} \tau_{\varepsilon}^{2}\right) \\
& =J_{\varepsilon}\left[\tilde{w}_{\varepsilon}, P_{\varepsilon}\right]+\mathrm{o}\left(\varepsilon^{5} e^{-\frac{\hat{d}_{\varepsilon}}{\varepsilon}}+\varepsilon^{3} e^{-3 \frac{\hat{d}_{\varepsilon}}{\varepsilon}}+\varepsilon^{6}\right)
\end{aligned}
$$

as $\varepsilon \rightarrow 0^{+}$.

Using a)-b) of Proposition 2.1 and (5.11) for $\varepsilon>0$ sufficiently small we get

$$
\begin{aligned}
& \int_{\Omega}\left|v_{\varepsilon}^{2}(-\Delta)^{-1}\left[v_{\varepsilon}^{2}\right] \mathrm{d} x-\tilde{w}_{\varepsilon, P_{\varepsilon}}^{2}(-\Delta)^{-1}\left[\tilde{w}_{\varepsilon, P_{\varepsilon}}^{2}\right]-4 \tau_{\varepsilon} \tilde{w}_{\varepsilon, P_{\varepsilon}} \phi_{\varepsilon}(-\Delta)^{-1}\left[\tilde{w}_{\varepsilon, P_{\varepsilon}}^{2}\right]\right| \mathrm{d} x \\
& =\mathrm{O}\left(\varepsilon^{3} \tau_{\varepsilon}^{2}\right),
\end{aligned}
$$

by which

$$
\begin{aligned}
J_{\varepsilon}\left[v_{\varepsilon}\right]= & J_{\varepsilon}\left[\tilde{w}_{\varepsilon, P_{\varepsilon}}\right]-\tau_{\varepsilon} \int_{\Omega} \mathcal{S}_{\varepsilon}\left[\tilde{w}_{\varepsilon, P_{\varepsilon}}\right] \phi_{\varepsilon} \mathrm{d} x \\
& -\int_{\Omega}\left(F\left(\tilde{w}_{\varepsilon, P_{\varepsilon}}+\tau_{\varepsilon} \phi_{\varepsilon}\right)-F\left(\tilde{w}_{\varepsilon, P_{\varepsilon}}\right)-\tau_{\varepsilon} f\left(\tilde{w}_{\varepsilon, P_{\varepsilon}}\right) \phi_{\varepsilon}\right) \mathrm{d} x \\
& +\tau_{\varepsilon}^{2} \frac{\varepsilon^{2}}{2} \int_{\Omega}\left|\nabla \phi_{\varepsilon}\right|^{2} \mathrm{~d} x+\frac{\tau_{\varepsilon}^{2}}{2} \int_{\Omega}\left|\phi_{\varepsilon}\right|^{2} \mathrm{~d} x+\mathrm{O}\left(\varepsilon^{3} \tau_{\varepsilon}^{2}\right) .
\end{aligned}
$$

Observe that $\left|F\left(\tilde{w}_{\varepsilon, P_{\varepsilon}}+\tau_{\varepsilon} \phi_{\varepsilon}\right)-F\left(\tilde{w}_{\varepsilon, P_{\varepsilon}}\right)-\tau_{\varepsilon} f\left(\tilde{w}_{\varepsilon, P_{\varepsilon}}\right) \phi_{\varepsilon}\right| \leq C \tau_{\varepsilon}^{2}\left|\phi_{\varepsilon}\right|^{2}$, while, by Lemma 3.4, $\mathcal{S}_{\varepsilon}\left[\tilde{w}_{\varepsilon, P_{\varepsilon}}\right]=\mathrm{o}\left(\tau_{\varepsilon} w_{\varepsilon, P_{\varepsilon}}^{1 / 4}\right) ;(5.11)$ leads to $J_{\varepsilon}\left[v_{\varepsilon}\right]=J_{\varepsilon}\left[\tilde{w}_{\varepsilon, P_{\varepsilon}}\right]+\mathrm{o}\left(\varepsilon^{3} \tau_{\varepsilon}^{2}\right)$. The choice of $\tau_{\varepsilon}$ allows us to conclude.

Step 2. $\frac{\hat{d}_{\varepsilon}}{\varepsilon|\log \varepsilon|} \rightarrow 1$ as $\varepsilon \rightarrow 0^{+}$and $\frac{\hat{d}_{\varepsilon}}{\varepsilon} \geq \log \frac{1}{\varepsilon}+\frac{1}{3} \log \log \frac{1}{\varepsilon}$ for $\varepsilon$ sufficiently small.

By inserting in (6.1) the estimates (4.2), (4.3) and (4.10) of Propositions 4.34.4 (taking into account that $\varepsilon^{3} e^{-3 \frac{\hat{d}_{\varepsilon}}{\varepsilon}}=\mathrm{o}\left(\frac{\varepsilon^{4}}{\hat{d}_{\varepsilon}} e^{-2 \frac{\hat{d}_{\varepsilon}}{\varepsilon}}\right)$ ), we obtain

$$
\begin{aligned}
J_{\varepsilon}^{*}= & I[w] \varepsilon^{3}+I_{2} \varepsilon^{5}-I_{3} \frac{\varepsilon^{6}}{\hat{d}_{\varepsilon}}+\varepsilon^{4}(1+\mathrm{o}(1)) B \frac{e^{-2 \frac{\hat{d}_{\varepsilon}}{\varepsilon}}}{4 \hat{d}_{\varepsilon}}+\mathrm{O}\left(\varepsilon^{4} \sqrt{\frac{\hat{d}_{\varepsilon}}{\varepsilon}} e^{-2 \frac{\hat{d}_{\varepsilon}}{\varepsilon}}\right) \\
& +\mathrm{o}\left(\varepsilon^{11 / 2}+\varepsilon^{5} e^{-\frac{\hat{d}_{\varepsilon}}{\varepsilon}}\right)
\end{aligned}
$$

as $\varepsilon \rightarrow 0^{+}$. First we will prove that $\lim \sup _{\varepsilon \rightarrow 0^{+}} \frac{\hat{d}_{\varepsilon}}{\varepsilon|\log \varepsilon|} \leq 1$. Assume by contradiction the existence of a sequence $\varepsilon_{n} \rightarrow 0^{+}$such that $\frac{\hat{d}_{\varepsilon_{n}}}{\varepsilon_{n}}>(1+\eta) \log \frac{1}{\varepsilon_{n}}$ for some $\eta>0$. In this case it is easy to show that the main part in the expansion on the right hand side of (6.2) is given by the first three terms, and the others are negligible, i.e.

$$
J_{\varepsilon_{n}}^{*} \geq I[w] \varepsilon_{n}^{3}+I_{2} \varepsilon_{n}^{5}-I_{3}(1+\mathrm{o}(1)) \frac{\varepsilon_{n}^{5}}{(1+\eta)\left|\log \varepsilon_{n}\right|},
$$


in contradiction with Corollary 5.2. Hence $\lim \sup _{\varepsilon \rightarrow 0^{+}} \frac{\hat{d}_{\varepsilon}}{\varepsilon|\log \varepsilon|} \leq 1$. Next we will prove that $\frac{\hat{d}_{\varepsilon}}{\varepsilon} \geq \log \frac{1}{\varepsilon}+\frac{1}{3} \log \log \frac{1}{\varepsilon}$ for small $\varepsilon$. Otherwise let $\varepsilon_{n} \rightarrow 0^{+}$be a sequence such that $\frac{\hat{d}_{\varepsilon_{n}}}{\varepsilon_{n}}<\log \frac{1}{\varepsilon_{n}}+\frac{1}{3} \log \log \frac{1}{\varepsilon_{n}}$. Hence we easily get $\varepsilon_{n}^{4} \sqrt{\frac{\hat{d}_{\varepsilon_{n}}}{\varepsilon_{n}}} e^{-2 \frac{\hat{d}_{\varepsilon_{n}}}{\varepsilon_{n}}}, \varepsilon_{n}^{5} e^{-\frac{\hat{d}_{\varepsilon_{n}}}{\varepsilon_{n}}}=$ $\mathrm{o}\left(\frac{\varepsilon_{n}^{4}}{\hat{d}_{\varepsilon_{n}}} e^{-2 \frac{\hat{d}_{n}}{\varepsilon_{n}}}\right)$ and (6.2) becomes

$$
J_{\varepsilon_{n}}^{*} \geq I[w] \varepsilon_{n}^{3}+I_{2} \varepsilon_{n}^{5}-I_{3} \frac{\varepsilon_{n}^{6}}{\hat{d}_{\varepsilon_{n}}}+\varepsilon_{n}^{4} B \frac{e^{-2 \frac{\hat{d}_{\varepsilon_{n}}}{\varepsilon_{n}}}}{8 \hat{d}_{\varepsilon_{n}}}+\mathrm{O}\left(\varepsilon_{n}^{11 / 2}\right) \text { as } \varepsilon \rightarrow 0^{+} .
$$

Set

$$
\rho_{n}(x)=-I_{3} \frac{\varepsilon_{n}^{2}}{x}+B \frac{e^{-2 x}}{8 x}, \quad x \geq \frac{\hat{d}_{\varepsilon_{n}}}{\varepsilon_{n}}
$$

and compute

$$
\rho_{n}^{\prime}(x)=I_{3} \frac{\varepsilon_{n}^{2}}{x^{2}}-B \frac{e^{-2 x}}{8 x^{2}}-B \frac{e^{-2 x}}{4 x}
$$

By taking the logarithm, $\rho_{n}^{\prime}(x) \geq 0$ in $\left(\frac{\hat{d}_{\varepsilon_{n}}}{\varepsilon_{n}},+\infty\right)$ implies

$$
x \geq \log \frac{1}{\varepsilon_{n}}+\frac{1}{2} \log x+\frac{1}{2} \log \left(\frac{1}{2 x}+1\right)+\frac{1}{2} \log \frac{B}{4 I_{3}} \geq \log \frac{1}{\varepsilon_{n}}+\frac{1}{2} \log \log \frac{1}{\varepsilon_{n}} .
$$

In particular, for $n$ sufficiently large, $\rho_{n}$ is decreasing in $\left(\frac{\hat{d}_{\varepsilon_{n}}}{\varepsilon_{n}}, \log \frac{1}{\varepsilon_{n}}+\frac{1}{3} \log \log \frac{1}{\varepsilon_{n}}\right)$, by which, proceeding as in Corollary 5.2,

$\rho_{n}\left(\frac{\hat{d}_{\varepsilon_{n}}}{\varepsilon_{n}}\right) \geq \rho_{n}\left(\log \frac{1}{\varepsilon_{n}}+\frac{1}{3} \log \log \frac{1}{\varepsilon_{n}}\right)=-I_{3} \frac{\varepsilon_{n}^{2}}{\left|\log \varepsilon_{n}\right|}+B(1+\mathrm{o}(1)) \frac{\varepsilon_{n}^{2}}{8\left|\log \varepsilon_{n}\right|^{5 / 3}}$.

Inserting this inequality in (6.3) we obtain

$$
J_{\varepsilon_{n}}^{*} \geq I[w] \varepsilon_{n}^{3}+I_{2} \varepsilon_{n}^{5}-I_{3} \frac{\varepsilon_{n}^{5}}{\left|\log \varepsilon_{n}\right|}+B(1+\mathrm{o}(1)) \frac{\varepsilon_{n}^{5}}{8\left|\log \varepsilon_{n}\right|^{5 / 3}}
$$

again in contradiction with Corollary 5.2.

Step 3. $\mathcal{H}\left(\Sigma_{P_{\varepsilon}}\right) \rightarrow \mathcal{H}_{0}=\max _{P \in \partial \Omega} \mathcal{H}(P)$.

In view of Step 2 all the error terms in the expansion (4.2), (4.11) and in (5.1) (with $d_{\varepsilon}=\hat{d}_{\varepsilon}$ ) are o $\left(\varepsilon^{6}\right)$; for example $\varepsilon^{4} \sqrt{\frac{\hat{d}_{\varepsilon}}{\varepsilon}} e^{-2 \frac{\hat{d}_{\varepsilon}}{\varepsilon}}=\mathrm{O}\left(\frac{\varepsilon^{6}}{|\log |^{1 / 6}}\right)=\mathrm{o}\left(\varepsilon^{6}\right)$. Hence 
we can write

$$
\begin{aligned}
I[w] \varepsilon^{3}+\varepsilon^{3} \alpha\left(\frac{\hat{d}_{\varepsilon}}{\varepsilon}\right) & +I_{2} \varepsilon^{5}-I_{3} \frac{\varepsilon^{6}}{\hat{d}_{\varepsilon}}-I_{4} \mathcal{H}\left(\Sigma_{P_{\varepsilon}}\right) \varepsilon^{6}+o\left(\varepsilon^{6}\right) \leq J_{\varepsilon}\left[v_{\varepsilon}\right] \\
& \leq I[w] \varepsilon^{3}+\varepsilon^{3} \alpha\left(\frac{\hat{d}_{\varepsilon}}{\varepsilon}\right)+I_{2} \varepsilon^{5}-I_{3} \frac{\varepsilon^{6}}{\hat{d}_{\varepsilon}}-I_{4} \mathcal{H}_{0} \varepsilon^{6}+o\left(\varepsilon^{6}\right)
\end{aligned}
$$

and Step 3 follows immediately.

Step 4. End of the proof.

By Corollary 3.3 and Proposition 5.4 we deduce Part (1) of Theorem 1.1. Parts (2) and (3) have been proved in Steps 2-3.

\section{References}

[1] P. W. BATES and J. SHI, Existence and instability of spike layer solutions to singular perturbation problems, J. Funct. Anal. 196 (2002), 211-264.

[2] P. W. BATES, E. N. DANCER and J. SHI, Multi-spike stationary solutions of the CahnHilliard equation in higher-dimension and instability, Adv. Differential Equations 4 (1999), 1-69.

[3] V. BENCI and D. FortunAto, An eigenvalue problem for the Schrödinger-Maxwell equations, Topol. Methods Nonlinear Anal. 11 (1998), 283-293.

[4] T. CAZENAVE and P. L. LiONS, Orbital stability of standing waves for some nonlinear Schrödinger equations, Comm. Math. Phys. 85 (1982), 549-561.

[5] C. C. CHEN and C. S. LIN, Uniqueness of the ground state solution of $\Delta u+f(u)=0$ in $\mathbb{R}^{N}, N \geq 3$, Comm. Partial Differential Equations 16 (1991), 1549-1572.

[6] G. M. Coclite and V. GeORGIEV, Solitary waves for Maxwell-Schrödinger equations, Electron. J. Differential Equations 94 (2004), 1-31.

[7] E. N. DANCER, Stable and finite Morse index solutions on $\mathbb{R}^{N}$ or on bounded domains with small diffusion. II, Indiana Univ. Math. J. 53 (2004), 97-108.

[8] E. N. DANCER and S. YAN, Effect of the domain geometry on the existence of multipeak solutions for an elliptic problem, Topol. Methods Nonlinear Anal. 14 (1999), 1-38.

[9] E. N. DANCER and S. YAN, Multipeak solutions for a singularly perturbed Neumann problem, Pacific J. Math. 189 (1999), 241-262.

[10] E. N. DANCER and S. YAN, Interior and boundary peak solutions for a mixed boundary value problem, Indiana Univ. Math. J. 48 (1999), 1177-1212.

[11] E. N. DANCER and S. YAn, Peak solutions for an elliptic system of Fitzhugh-Nagumo type, Ann. Scuola Norm. Sup. Pisa Cl. Sci. (5) 2 (2003), 679-709.

[12] E. N. DANCER and S. YAN, On the profile of the changing-sign mountain pass solutions for an elliptic problem, Trans. Amer. Math. Soc. 354 (2002), 3573-3600.

[13] T. D'APRILE and D. Mugnai, Existence of solitary waves for the nonlinear KleinGordon Maxwell and Schrödinger-Maxwell equations, Proc. Roy. Soc. Edinburgh Sect. A 134 (2004), 893-906.

[14] T. D'APRILE and J. WEI, Boundary concentration in radial solutions for a system of semilinear elliptic equations, J. London Math. Soc. (2), to appear.

[15] T. D' APRILE and J. WEI, Clustered solutions around harmonic centers to a coupled elliptic system, Ann. Inst. H. Poincaré Anal. Non Linéaire, to appear. 
[16] T. D'APRILE and J. WEI, On bound states concentrating on spheres for the MaxwellSchrödinger equation, SIAM J. Math. Anal. 37 (2005), 321-342.

[17] T. D' APRILE and J. WeI, Standing waves in the Maxwell-Schrödinger equation and an optimal configuration problem, Calc. Var. Partial Differential Equations 25 (2006), 105137.

[18] M. Del PINo and P. Felmer, Spike-layered solutions of singularly perturbed elliptic problems in a degenerate setting, Indiana Univ. Math. J. 48 (1999), 883-898.

[19] M. Del Pino, P. Felmer and J. WeI, On the role of mean curvature in some singularly perturbed Neumann problems, SIAM J. Math. Anal. 31 (1999), 63-79.

[20] M. J. EsteBAN and P. L. LiONS, Existence and non-existence results for semilinear elliptic problems in unbounded domains, Proc. Roy. Soc. Edinburgh Sect. A 93 (1982), 1-14.

[21] H. EGNELL, Asymptotic results for finite energy solutions of semilinear elliptic equations, J. Differential Equations, 98 (1992), 34-56.

[22] L. C. Evans "Partial Differential Equations", American Mathematical Society, Providence, Rhode Island, 1998.

[23] B. GidAs, W. M. NI and L. NiRENBERG, Symmetry of positive solutions of nonlinear elliptic equations in $\mathbb{R}^{N}$, In: "Mathematical Analysis and Applications", Part A, N. Nachbin (ed.), Adv. Math. Suppl. Stud., Vol. 7, 1981, 369-402.

[24] D. Gilbarg and N. S. Trudinger, "Elliptic Partial Differential Equations of Second Order", Springer Verlag Berlin Heidelberg, 2001.

[25] M. GROSSI and A. PISTOIA, On the effect of critical points of distance function in superlinear elliptic problems, Adv. Differential Equations 5 (2000), 1397-1420.

[26] M. Grossi, A. PISTOIA and J. WeI, Existence of multipeak solutions for a semilinear Neumann problem via nonsmooth critical point theory, Calc. Var. Partial Differential Equations 11 (2000), 143-175.

[27] C. GUI and J. WEI, Multiple interior spike solutions for some singular perturbed Neumann problems, J. Differential Equations 158 (1999), 1-27.

[28] C. GUI and J. WEI, On multiple mixed interior and boundary peak solutions for some singularly perturbed Neumann problems, Canad. J. Math. 52 (2000), 522-538.

[29] C. GUI, J. WEI and M. WINTER, Multiple boundary peak solutions for some singularly perturbed Neumann problems, Ann. Inst. H. Poincaré Anal. Non Linéaire 17 (2000), 249289.

[30] L. L. Helms, "Introduction to Potential Theory", John Wiley \& sons Inc., New York, 1969.

[31] M. K. Kwong, Uniqueness of positive solutions of $\Delta u-u+u^{p}=0$ in $\mathbb{R}^{N}$, Arch. Ration. Mech. Anal. 105 (1991), 243-266.

[32] Y. Y. LI, On a singularly perturbed equation with Neumann boundary conditions, Commun. Partial Differential Equations 23 (1998), 487-545.

[33] Y.-Y. LI and L. NiRENBERG, The Dirichlet problem for singularly perturbed elliptic equations, Comm. Pure Appl. Math. 51 (1998), 1445-1490.

[34] W. M. Ni and I. TAKAGI, Locating the peaks of least energy solutions to a semilinear Neumann problem, Duke Math. J. 70 (1993), 247-281.

[35] W. M. Ni and I. TAKAGI, On the shape of least energy solutions to a semi-linear Neumann problem, Comm. Pure Appl. Math. 44 (1991), 819-851.

[36] W. M. NI, I. TAKAGI and J. WEI, On the location and profile of spike-layer solutions to singularly perturbed semilinear Dirichlet problems: intermediate solutions, Duke Math. J. 94 (1998), 597-618.

[37] W. M. NI and J. WEI, On the location and profile of spike-layer solutions to singularly perturbed semilinear Dirichlet problems, Comm. Pure Appl. Math. 48 (1995), 723-761.

[38] D. RuIZ, Semiclassical states for coupled Schrödinger-Maxwell equations: concentration around a sphere, Math. Models Methods Appl. Sci. 15 (2005), 141-164.

[39] J. WEI, On the boundary spike layer solutions of a singularly perturbed semilinear Neumann problem, J. Differential Equations 134 (1997), 104-133. 
[40] J. WEI, On the construction of single-peaked solutions to a singularly perturbed semilinear Dirichlet problem, J. Differential Equations 129 (1996), 315-333.

[41] J. WEI, Conditions for two-peaked solutions of singularly perturbed elliptic equations, Manuscripta Math. 96 (1998), 113-131.

[42] X. P. ZHU, Multiple entire solutions of a semilinear elliptic equation, Nonlinear Analysis TMA 12 (1998), 1297-1316.

Dipartimento di Matematica

Università degli Studi di Roma "Tor Vergata"

Via della Ricerca Scientifica, 1

00133 Roma, Italy

daprile@mat.uniroma2.it

Department of Mathematics

The Chinese University of Hong Kong

Shatin, Hong Kong

wei@math.cuhk.edu.hk 